\title{
Spectral evolution of Nova (V1494) Aql and its high velocity jets ${ }^{\star}$
}

\author{
T. Iijima ${ }^{1}$ and H. H. Esenoglu ${ }^{2,3}$ \\ 1 Astronomical Observatory of Padova, Asiago Section, Osservatorio Astrofisico, 36012 Asiago (Vi), Italy \\ 2 Astronomy and Space Science Department of Istanbul University, University Observatory, 34452, University, Istanbul, \\ Turkey \\ e-mail: esenoglu@istanbul.edu.tr \\ 3 Istanbul University Observatory Research and Application Center, 34452, University, Istanbul, Turkey
}

Received 28 January 2003 / Accepted 21 March 2003

\begin{abstract}
Spectral evolution of the fast nova V1494 Aql was monitored soon after its discovery in December 1999 to September 2000. The first spectra showed prominent emission lines of H I and Fe II, while He I was seen in absorption. The radial velocities of the absorption components of H I, He I and N II rapidly increased (in the negative sense) during the early decline stage, while those of Fe II remained nearly constant. When a new spectrum was taken on February 6, 2000 after the seasonal interruption, this nova was in the transition stage. The spectra in the transition stage showed emission lines of $\mathrm{HI}$, He I, He II, N II, N III, Si II, [N II], [O I], [O III], [Fe II], [Fe VI], [Ca V] etc., hence the emission lines of Fe II had disappeared. A quasi-periodic oscillation of luminosity with a time scale of about $16.5 \pm 1$ days and a mean amplitude of about 1.2 mag in $V$ band was seen from February to the middle of April 2000. The emission lines of He II and [Ca V] disappeared around a light maximum of the oscillation, while the emission lines of N II and N III strengthened. At the same time high velocity ( -2900 and $+2830 \mathrm{~km} \mathrm{~s}^{-1}$ ) broad emission wings of $\mathrm{H}$ I lines appeared, which suggest an ejection of high velocity jets. The excitation state increased throughout the nebular stage. The last spectra taken in September 2000 showed highly excited emission lines up to $[\mathrm{Fe} \mathrm{VII}]$ and $[\mathrm{Fe} \mathrm{X}] \lambda 6374.5$.

The interstellar extinction is estimated as $E(B-V)=0.6 \pm 0.1$ from the equivalent widths of the interstellar absorption components of $\mathrm{Na}$ I D1 and D2. Using this result, the distance to the nova is estimated as $1.6 \pm 0.2 \mathrm{kpc}$. The mass and the helium abundance of the ejecta are estimated as $6.2 \pm 1.4 \times 10^{-5} M_{\odot}$ and $N(\mathrm{He}) / N(\mathrm{H})=0.13 \pm 0.01$, respectively. The electron density of the ejecta decreased as $N_{\mathrm{e}} \propto t^{-0.8}$ during the nebular stage, where $t$ is time from light maximum. This low decline rate suggests that the ejecta had a ring like shape as well a large mass loss which may have continued throughout the nebular stage.
\end{abstract}

Key words. stars: individual: V1494 Aql - novae, cataclysmic variables - ISM: general

\section{Introduction}

V1494 Aql (Nova Aql 1999-II) was discovered by Pereira (1999) on December 1, 1999 as a star of $m_{\text {Vis. }} \cong 6.0$. The luminosity and time of light maximum were estimated as $m_{\mathrm{V}} \cong 4.0$ on December 3.4 UT, 1999, JD 2451515.9 , by Kiss \& Thomson (2000). Early spectral evolution was reported by Kiss \& Thomson (2000) and Anupama et al. (2001). Kiss \& Thomson (2000) obtained the decline rates of the luminosity $t_{2}=6.6 \pm 0.5$ days and $t_{3}=16 \pm 0.5$ days, and estimated the absolute magnitude at maximum $M_{\mathrm{V}}(\max )=-8.8 \pm 0.2$. They obtained a distance to the nova $D=3.6 \pm 0.3 \mathrm{kpc}$. This distance, however, should be an upper limit because the effect of the interstellar extinction was not taken into account. Kawabata et al. (2001) carried out some spectro-polarimetric observations

Send offprint requests to: T. Iijima,

e-mail: iijima@astras.pd.astro.it

$\star$ Table 1 is only available in electronic form at http://www. edpsciences.org and suggested that the ejecta were not spherically symmetric. Many coronal emission lines were detected by infrared spectroscopy carried out in July 2000 (Venturini et al. 2000) and July 2001 (Rudy et al. 2001).

The monitoring of spectral evolution of this nova in our observatory started soon after the announcement of discovery. The first spectra were taken on December 5, 1999, that is 2.3 days after maximum, then high and medium dispersion spectra were taken until September 16, 2000. In this paper, we report the spectral evolution of this nova in the early decline, transition, and nebular stages. The physical properties of the ejecta are estimated using the intensities of the emission lines in the nebular stage. The high velocity jets observed in the transition stage are also reported.

\section{Observation}

High dispersion spectra covering the range $\lambda \lambda 4350-6900 \AA$ with a resolution of $\lambda / \Delta \lambda \cong 8000$ were taken with an 
Echelle spectrograph mounted on the $182 \mathrm{~cm}$ telescope at the Mount Ekar station of the Astronomical Observatory of Padova. Medium dispersion spectra were taken with a Boller \& Chivens grating spectrograph mounted on the $122 \mathrm{~cm}$ telescope of the Astrophysical Observatory of Asiago of the University of Padova. The resolution is $\lambda / \Delta \lambda \cong 800$ with a grating of 600 lines $\mathrm{mm}^{-1}$. The reductions of the spectra were carried out using the standard tasks of the NOAO IRAF package at Asiago Observatory. The spectrophotometric calibrations were made using the spectra of standard stars taken in the same nights. HR 7596 (58 Aql) was used for the calibrations of the high dispersion spectra and Kopff 27 for the medium dispersion spectra. A $\log$ of the observations is given in Table 1, where UT is the universal time at the start of the exposure and "days" is number of days from light maximum.

\section{Interstellar extinction and the distance to the nova}

The effect of interstellar extinction was not taken into account in the previous works of this nova, because no interstellar absorption line was detected in their low dispersion spectra (Kiss \& Thomson 2000; Anupama et al. 2001). The interstellar absorption components of Na I D1 and D2, however, are clearly seen on our high dispersion spectra (see, e.g. Figs. 11-13). The mean equivalent widths of D1 and D2 are $0.46 \pm 0.01$ and $0.55 \pm 0.01 \AA$, respectively. Using the doubleratio method of Münch (1968), the column density of sodium atoms is estimated as $\log (N(\mathrm{Na}) L)=13.15 \pm 0.1$ atoms cm $\mathrm{cm}^{-2}$ from these equivalent widths, where $L$ is the length of the line of sight in $\mathrm{cm}$. The amount of the interstellar extinction may be $E(B-V)=0.6 \pm 0.1$ and $A_{\mathrm{V}}=1.8 \pm 0.3$, where we adopted the chemical abundance of the interstellar medium, $\log (N(\mathrm{Na}) / N(\mathrm{H}))=-8.4($ Cohen 1975$)$, and the relation between the column density of hydrogen atom and the interstellar extinction, $N(\mathrm{H}) L / E(B-V)=6.2 \times 10^{21}$ atoms $\mathrm{cm}^{-2} \mathrm{mag}^{-1}$ (Jenkins \& Savage 1974). The observed spectra are, therefore, dereddened assuming $E(B-V)=0.6$.

If we add the effect of the interstellar extinction, $A_{\mathrm{V}}=1.8$, to the result of Kiss \& Thomson (2000), the distance to the nova should be $1.6 \pm 0.2 \mathrm{kpc}$.

\section{Early decline stage}

\subsection{Spectral features}

Figure 1 shows the light curve of V1494 Aql collected from VSNET. This nova showed a fast fading in December 1999. Our first spectra were taken on December 5 with the Boller \& Chivens spectrograph, then successive observations in the early decline stage were made on December 6 and 17.

Figures 2-4 show tracings of our first spectra, where the unit of the ordinate is $10^{-10} \mathrm{erg} \mathrm{cm}^{-2} \mathrm{~s}^{-1} \AA^{-1}$. Most prominent emission lines are of $\mathrm{H} \mathrm{I}$ and Fe II, whereas He I and some $\mathrm{N}$ II lines are seen as absorptions. The weak emission line at $\lambda 6165.4 \AA$ could be a blend of O I $\lambda 6157.0$ and N II $\lambda 6167.8$ (Fig. 4). The emission components of $\mathrm{H} \alpha, \mathrm{H} \beta$ and $\mathrm{H} \gamma$ show single peak profiles, while $\mathrm{H} \delta$ and some Fe II lines show saddle

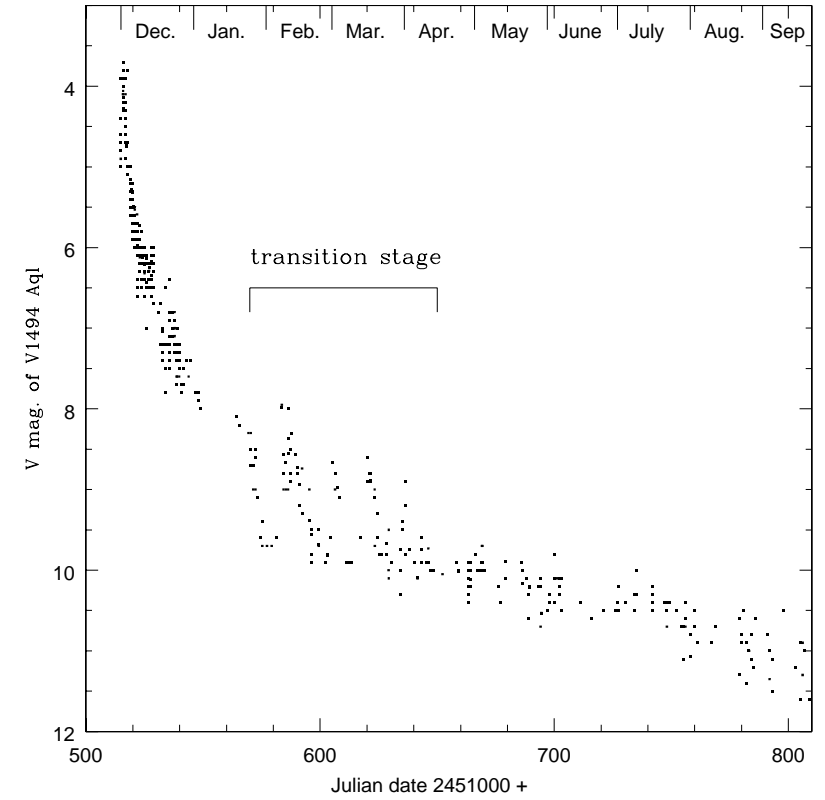

Fig. 1. Light curve of V1494 Aql collected from VSNET (http://www. kusastro.kyoto-u.ac.jp/vsnet/index.html).

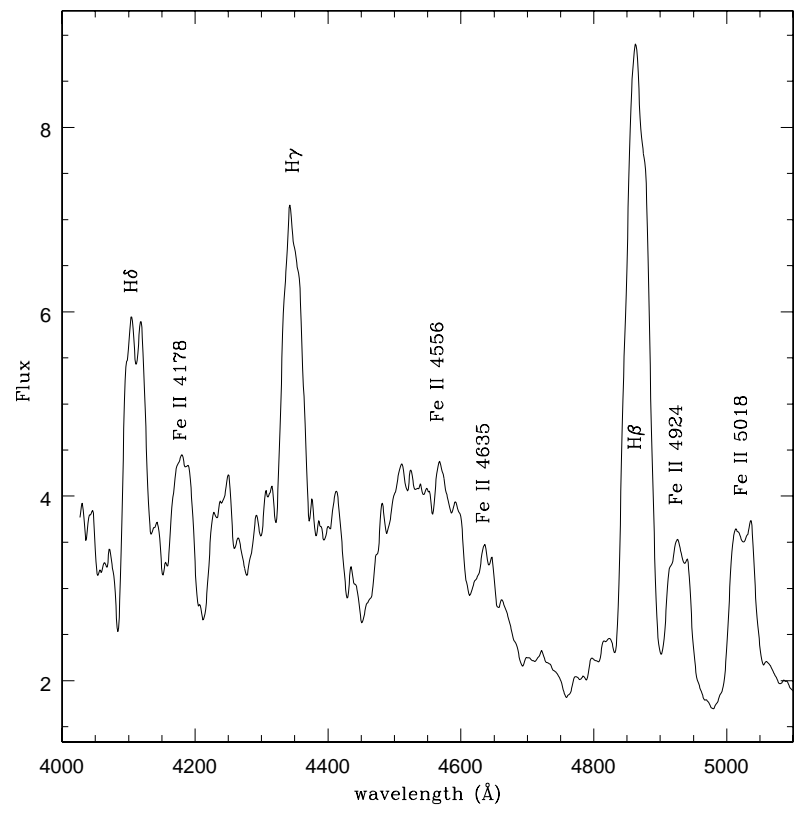

Fig. 2. A spectrum of V1494 Aql on Dec. 5, 1999. The unit of the ordinate is $10^{-10} \mathrm{erg} \mathrm{cm}^{-2} \mathrm{~s}^{-1} \AA^{-1}$.

shaped profiles. Some H I and Fe II lines are flanked by P Cygni type absorption components. The absorption components of $\mathrm{H} \beta$ and $\mathrm{H} \gamma$ are very weak and that of $\mathrm{H} \alpha$ is not detected in our medium dispersion spectra.

The spectral features on December 6 were effectively the same as those on December 5, while large changes were noticed on December 17. Figures 5-7 show tracings of the spectra on December 17. A tracing of the spectrum multiplied by ten is over-plotted as a dotted line in Fig. 7 to reveal the weak spectral features. In contrast to the earlier spectra, $\mathrm{H} \alpha$ and $\mathrm{H} \beta$ show profiles with well separated two peaks and a weak central peak. The emission components of $\mathrm{H} \beta$ and the higher member 


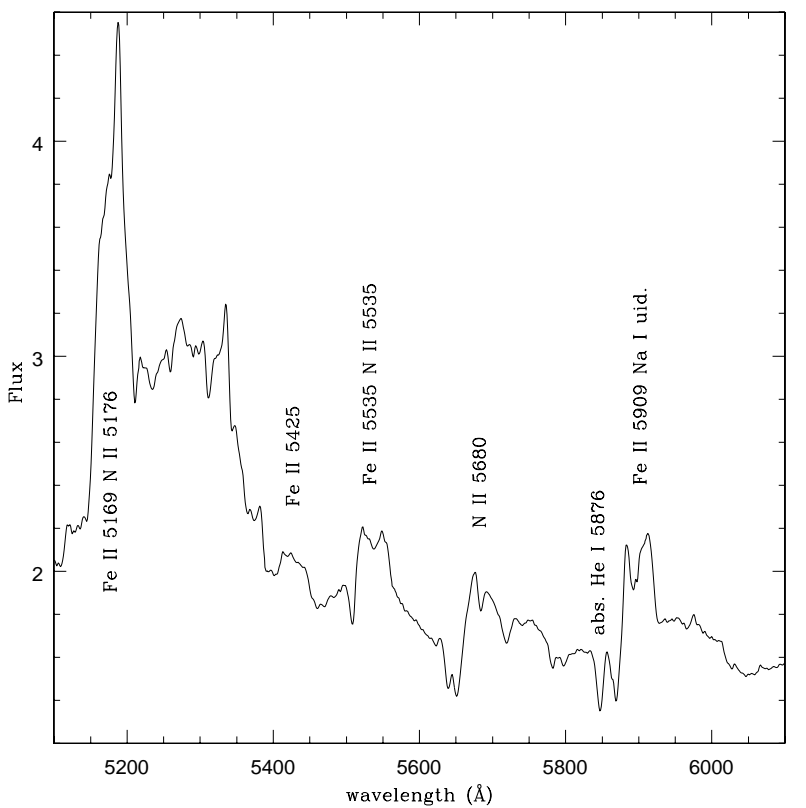

Fig. 3. A spectrum of V1494 Aql on Dec. 5, 1999. The unit of the ordinate is $10^{-10} \mathrm{erg} \mathrm{cm}^{-2} \mathrm{~s}^{-1} \AA^{-1}$.

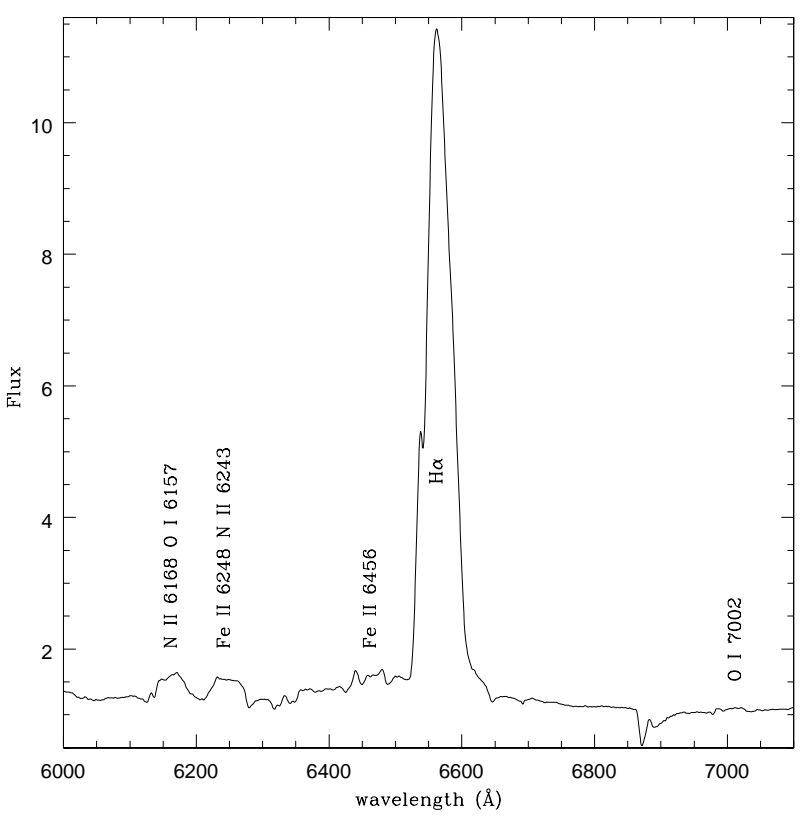

Fig. 4. A spectrum of V1494 Aql on Dec. 5, 1999. The unit of the ordinate is $10^{-10} \mathrm{erg} \mathrm{cm}^{-2} \mathrm{~s}^{-1} \AA^{-1}$.

of Balmer series are flanked by P Cygni type absorption components. The blue-ward emission peaks of $\mathrm{H} \gamma$ and $\mathrm{H} \delta$ seem to be cut out by the absorption components. A weak trace of [O I] $\lambda 5577.4$ is seen on the spectrum on December 17 (Fig. 6). This line was not seen on any other earlier nor later spectra.

An absorption line is seen at $\lambda 4608 \AA$ on the spectrum taken on December 17 (Fig. 5). If this line is due to N III $\lambda 4640.6$, its radial velocity should be $-2110 \mathrm{~km} \mathrm{~s}^{-1}$. This velocity, however, is too low with respect to those of the other N II and He I lines (see, Sect. 4.3). This

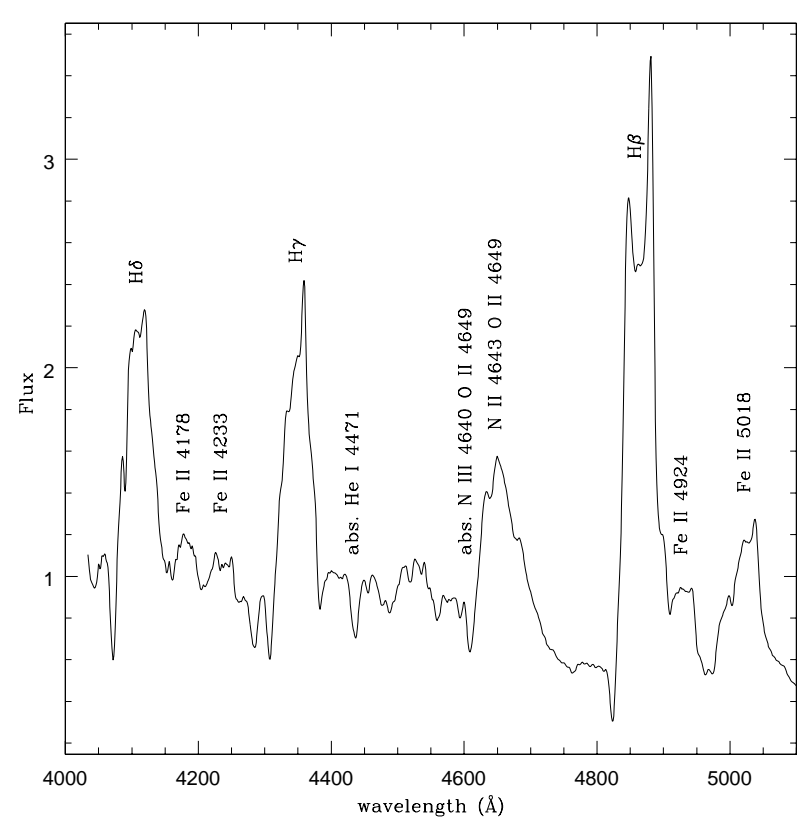

Fig. 5. A spectrum of V1494 Aql on Dec. 17, 1999. The unit of the ordinate is $10^{-10} \mathrm{erg} \mathrm{cm}^{-2} \mathrm{~s}^{-1} \AA^{-1}$.

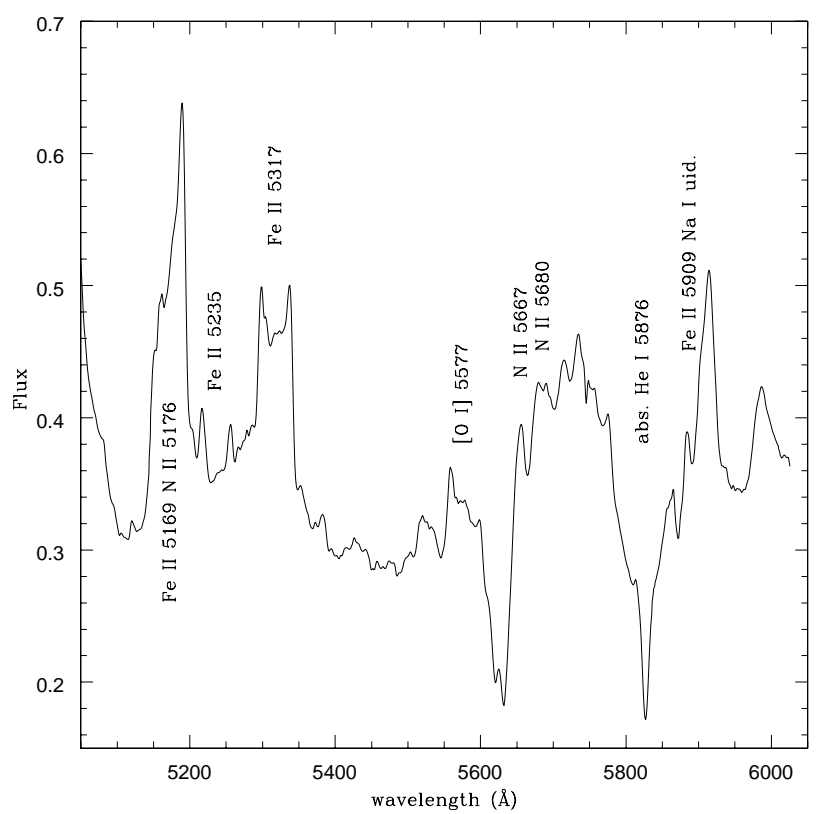

Fig. 6. A spectrum of V1494 Aql on Dec. 17, 1999. The unit of the ordinate is $10^{-10} \mathrm{erg} \mathrm{cm}^{-2} \mathrm{~s}^{-1} \AA^{-1}$.

absorption line may be due to a blend of N III $\lambda 4640.6$, N II $\lambda 4643.1$ and O II $\lambda 4649.1$.

The changes of the profiles of $\mathrm{H} \alpha$ also have been reported in previous works (Kiss \& Thomson 2000; Anupama et al. 2001). The observations made by Anupama et al. (2001) show that the profile of $\mathrm{H} \alpha$ largely changed between December 10 and 16 . When the profiles of the H I lines changed, their widths significantly increased as shown in Table 2.

A weak emission line is seen at $\lambda 7105 \AA$ on the spectrum on December 17 (Fig. 7). This line was not observed in any previous novae or nova-like objects, at far as we know. Its laboratory wavelength may be $7105 \pm 2.5 \AA$. Here, we tentatively 


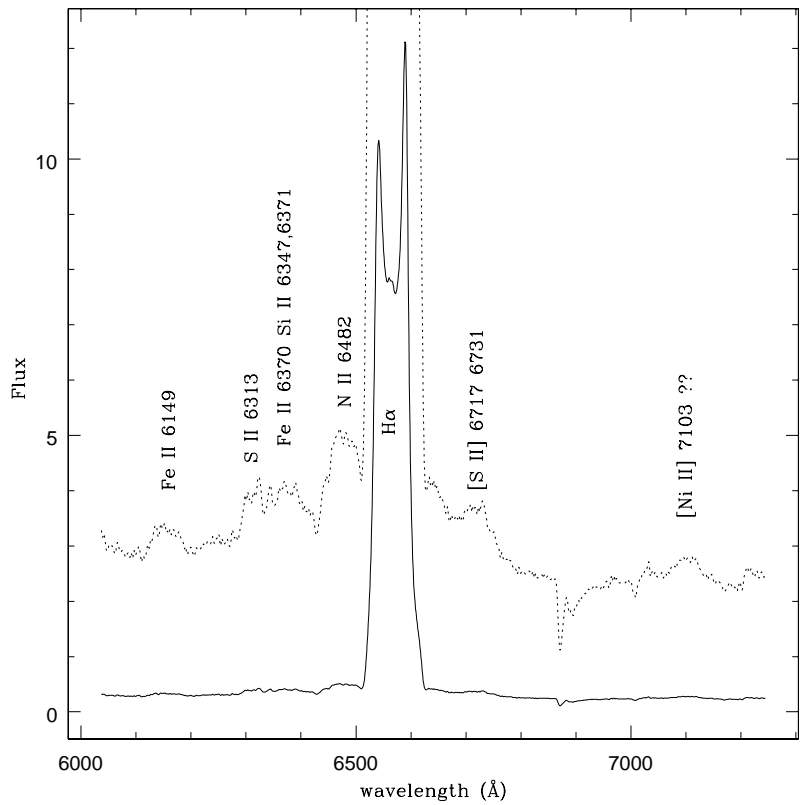

Fig. 7. A spectrum of V1494 Aql on Dec. 17, 1999. The unit of the ordinate is $10^{-10} \mathrm{erg} \mathrm{cm}^{-2} \mathrm{~s}^{-1} \AA^{-1}$. The dotted line shows a tracing of the spectrum multiplied by ten.

identify it as [Ni II] $13 \mathrm{~F} \lambda 7102.8$ (Moore 1959), but no other line of the same ion, e.g. [Ni II] 14F 15275.8 , was detected in the spectra. Further works are necessary for the identification of this line.

\subsection{Balmer decrement}

Intensities after the correction of the interstellar extinction by $E(B-V)=0.6$ of some selected emission lines in December 1999 are given in Table 2. The selected lines are relatively free from blending and are not necessarily stronger than the others. The intensity ratio of $\mathrm{H} \alpha / \mathrm{H} \beta$ and the $F W H M$ of $\mathrm{H} \beta$ are also presented. The observational errors in the intensities are about $\pm 10 \%$ and those in the $F W H M$ of $\mathrm{H} \beta$ are about $\pm 50 \mathrm{~km} \mathrm{~s}^{-1}$. Intensities affected by larger observational errors are denoted by a colon.

Figure 8 shows the variation of the intensity ratio $\mathrm{H} \alpha / \mathrm{H} \beta$, where the results in the transition and nebular stages are also plotted. The theoretical ratio of $\mathrm{H} \alpha / \mathrm{H} \beta$ in Case B recombination should be found in the range of 2.6-3.0 in various conditions of electron temperature and density (Hummer \& Storey 1987). As seen in Fig. 8 and Table 2, however, the ratio $\mathrm{H} \alpha / \mathrm{H} \beta$ of the present nova was lower than the theoretical values on December 5 and 6. The ratio suddenly increased afterwards and became much higher than the theoretical one on December 17. At the same time, the profiles of $\mathrm{H} \alpha$ and $\mathrm{H} \beta$ significantly changed and the width of $\mathrm{H} \beta$ increased, as reported in the previous sub-section. The high intensity ratio continued until April 2000, then gradually decreased (see also Sect. 6).

The large ratios of $\mathrm{H} \alpha / \mathrm{H} \beta$ on December 17 and later may not have been due to an ejection of obscure matter, because a deep light minimum such as of DQ Her (e.g. Martin 1989) was not seen in the light curve of this nova (Fig. 1). Netzer (1975) called attention to the effect of self-absorption of Lyman
Table 2. Intensities of selected emission lines in the early decline stage corrected by $E(B-V)=0.6$.

\begin{tabular}{llrrr}
\hline \hline $\begin{array}{l}\lambda_{\text {center }} \\
(\mathrm{A})\end{array}$ & Elements & Dec. 5 & $\begin{array}{r}I(\lambda) \\
\text { Dec. 6 }\end{array}$ & Dec. 17 \\
\hline 4108 & H $\delta$ & 75 & 80 & 46 \\
4180 & Fe II 4179 & 36 & 42 & 8.0 \\
4345 & H $\gamma$ & 107 & 115 & 51 \\
4637 & Fe II 4635 & 33 & 27 & $6:$ \\
4647 & N II 4643, O II 4649 & & & 33 \\
4863 & H $\beta$ & 231 & 219 & 101 \\
4928 & Fe II 4924 & 55 & 47 & 16 \\
5022 & Fe II 5018 & 71 & 64 & 30 \\
5180 & Fe II 5169, N II 5176 & 74 & 60 & 11 \\
5319 & Fe II 5317 & & & 8.4 \\
5537 & Fe II 5535, N II 5535 & 14 & 11 & \\
5576 & [O I] 5577.4 & & & 2.5 \\
5902 & Fe II 5909, Na I, uid. & 16 & 12 & 4.6 \\
6154 & Fe II 6149 & & & 2.4 \\
6164 & N II 6168, O I 6157 & 15 & 10 & \\
6247 & N II 6243, Fe II 6248 & 15 & 10 & $0.5:$ \\
6316 & S II 6313 & & & 3.3 \\
6568 & H $\alpha$ & 411 & 429 & 607 \\
6726 & [S II] 6717, 6731 & & & 2.7 \\
7006 & O I 7002 & 2.3 & 0.9 & \\
7105 & [Ni II] 7103? & & & 1.1 \\
\hline & H $\alpha / H \beta$ & 2.0 & 6.0 \\
& FWHM of H $\beta$ & 2420 & 2460 & 3120 \\
\hline
\end{tabular}

Unit of $I(\lambda)$ is $10^{-10} \mathrm{erg} \mathrm{cm}^{-2} \mathrm{~s}^{-1}$ and that of $F W H M$ is $\mathrm{km} \mathrm{s}^{-1}$.

and Balmer photons in high density nebulosity. Netzer (1975) showed that the ratio $\mathrm{H} \alpha / \mathrm{H} \beta$ from high density nebulosity are

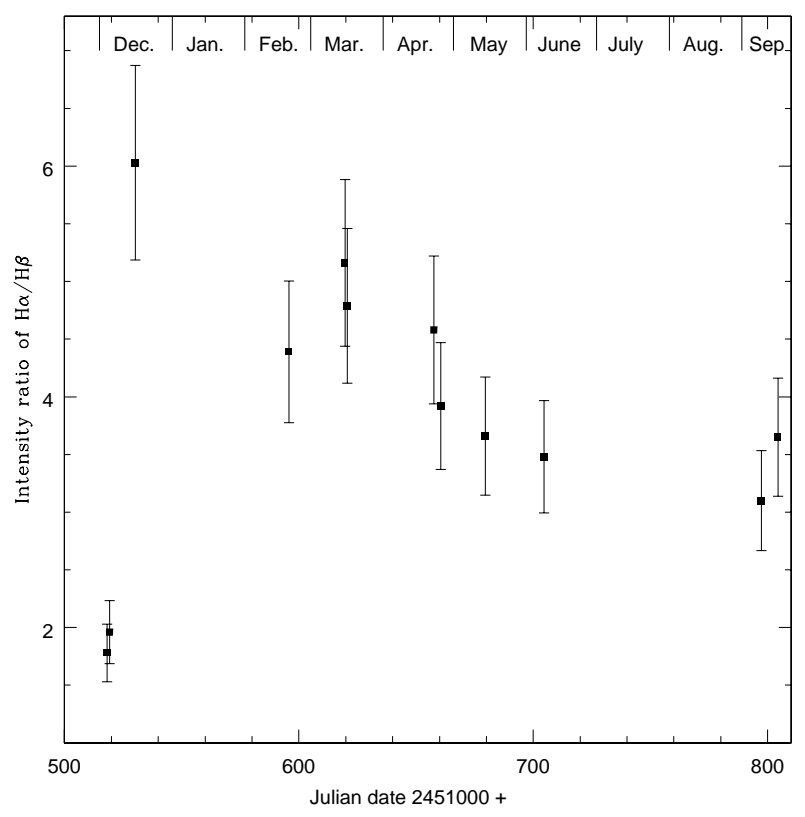

Fig. 8. Intensity ratio of $\mathrm{H} \alpha / \mathrm{H} \beta$. 
Table 3. Radial velocities of selected absorption lines in the early decline stage.

\begin{tabular}{lrrr}
\hline \hline Elements & \multicolumn{3}{c}{ Radial velocity in km s } \\
& Dec. 5 & Dec. 6 & Dec. 17 \\
\hline $\mathrm{H} \delta$ & -1420 & -1460 & -2320 \\
$\mathrm{H} \gamma$ & -1410 & -1520 & -2400 \\
Fe II 4403 & -1520 & -1490 & -1450 \\
$\mathrm{He}$ I 4471 & -1490 & -1580 & -2470 \\
Fe II 26, 4580 & -1570 & -1530 & -1470 \\
$\mathrm{H} \beta$ & & & -2450 \\
Fe II 49, 5235 & -1460 & -1450 & -1530 \\
Fe II 41, 5284 & -1520 & -1500 & \\
Fe II 48, 5338 & -1580 & -1540 & \\
Fe II 55, 5535 & -1540 & -1550 & \\
N II 3, 5667 & -1570 & -1650 & -2530 \\
N II 3, 5680 & -1620 & -1750 & -2590 \\
N II 3, 5711 & -1510 & -1660 & -2480 \\
N II 9, 5747 & -1540 & -1620 & -2450 \\
He I 5876 & -1560 & -1690 & -2560 \\
Na I D1+D2 & & -1660 & \\
O I 10, 6157 & -1610 & -1670 & -2240 \\
N II 36,60 6168 & -1600 & -1550 & -2520 \\
$\langle$ Fe II $\rangle$ & $-1530 \pm 40$ & $-1510 \pm 40$ & $-1480 \pm 60$ \\
$\langle$ He I+N II $\rangle$ & $-1560 \pm 40$ & $-1640 \pm 40$ & $-2510 \pm 40$ \\
\hline uid. & & 4454.7 & 4454.4 \\
uid. & 5868.3 & 5868.1 & 5870.5 \\
\hline
\end{tabular}

uid.: wavelength of unidentified absorption line in $\AA$.

found in the range from 1.28 to 19.89 owing to the effect of self-absorption.

When the optical depths of Lyman $\alpha\left(\tau_{\alpha}\right)$ and $\mathrm{H} \alpha\left(\tau_{\mathrm{H} \alpha}\right)$ are very high, e.g. $\tau_{\alpha}=10^{4}$ and $\tau_{\mathrm{H} \alpha}>300$, we have low ratios of $\mathrm{H} \alpha / \mathrm{H} \beta$ similar to those found on December 5 and 6. The intensity ratios higher than 4.0 are found in some conditions of higher optical depth of Lyman $\alpha$ and/or lower optical depth of $\mathrm{H} \alpha$ (Netzer 1975). It may be very likely that the optical depths of Lyman $\alpha$ and $\mathrm{H} \alpha$ were very high in the earliest stage, and the effect of self-absorption was important. The large variations of the ratio $\mathrm{H} \alpha / \mathrm{H} \beta$ could be explained with this effect. A similar variation of the ratio $\mathrm{H} \alpha / \mathrm{H} \beta$ was observed also in Nova (V1500) Cyg 1975 (Ferland 1978).

\subsection{Acceleration of ejecta}

Radial velocities of selected absorption lines are given in Table 3, where $\langle\mathrm{Fe}$ II $\rangle$ is the mean radial velocity of Fe II lines and $\langle\mathrm{He} \mathrm{I}+\mathrm{N}$ II $\rangle$ is that of He I and N II lines. The observational errors in the individual measurements are about $\pm 100 \mathrm{~km} \mathrm{~s}^{-1}$. The radial velocities are given in the frame of the nova system of which the receding velocity is estimated as $+46.3 \pm$ $0.5 \mathrm{~km} \mathrm{~s}^{-1}$ measuring the line center of the emission lines of [O III] and [N II] in the nebular stage. An absorption line at $\lambda 6125 \AA$ on December 5 is identified as the O I multiplet No. 10 (Moore 1959). The weighted mean of the wavelengths of the three contiguous lines of the multiplet is $\lambda 6157.03 \AA$.

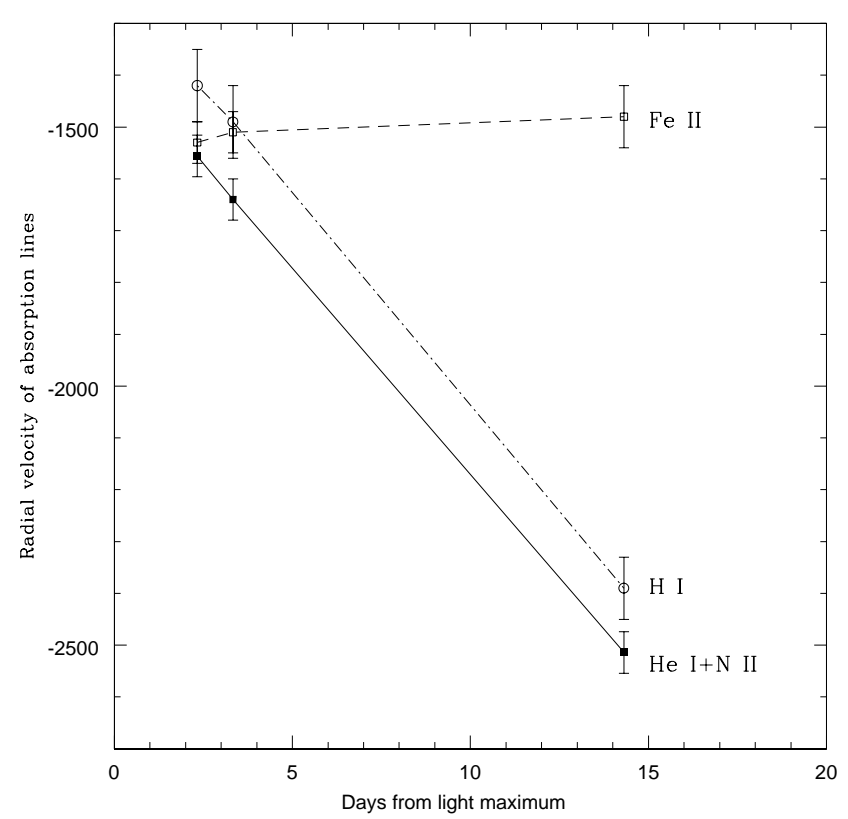

Fig. 9. Radial velocities of the absorption components of $\mathrm{H} \mathrm{I,} \mathrm{He} \mathrm{I,}$ $\mathrm{N}$ II and Fe II lines.

Figure 9 shows the mean radial velocities of the absorption components of H I, Fe II, and He I+N II. The radial velocities of $\mathrm{H} \mathrm{I}, \mathrm{He}$ I and N II rapidly increased (in negative sense) during the early decline stage. On the other hand, those of Fe II remained nearly constant or slightly decreased.

Increasing outflow velocity has been observed in many previous novae (e.g. McLaughlin 1960). This phenomenon may represent the increasing of escape velocity due to the shrink of the photosphere (see, e.g. the model of Kato \& Hachisu 1994). The absorption lines of Fe II might have been due to a remnant of the spectrum at light maximum.

Kiss \& Thomson (2000) estimated the expansion velocity of the ejecta as $2000 \mathrm{~km} \mathrm{~s}^{-1}$ from their observations on December 8 , but the outflow velocity largely increased after their observations. The mean radial velocity of He I and N II on December 17 was $-2510 \mathrm{~km} \mathrm{~s}^{-1}$, and it seems that the velocity was still increasing (in negative sense) at that time. The outflow velocity may have increased up to about $2900 \mathrm{~km} \mathrm{~s}^{-1}$, because as shown later high velocity jets having radial velocities of about $\pm 2900 \mathrm{~km} \mathrm{~s}^{-1}$ were observed in the transition stage (Sect. 5.2).

\subsection{Unidentified absorption lines}

There were two unidentified absorption lines in the spectra of the early decline stage. The wavelengths of the lines are given in Table 3. Since the wavelengths did not change so much, these lines may be related to the group of Fe II. If the radial velocities of these lines were the same as the mean of Fe II lines, their laboratory wavelengths should be $\lambda 4476.2 \pm 1.0$ and $\lambda 5897.7 \pm$ $1.0 \AA$, respectively. We are, however, not able to find good candidates for these lines in the table of Moore (1959).

One possible interpretation for these lines may be that the former line is identified as $\mathrm{He}$ I $\lambda 4471$ blue-shifted by $-1130 \mathrm{~km} \mathrm{~s}^{-1}$ on December 6 and $-1150 \mathrm{~km} \mathrm{~s}^{-1}$ on 


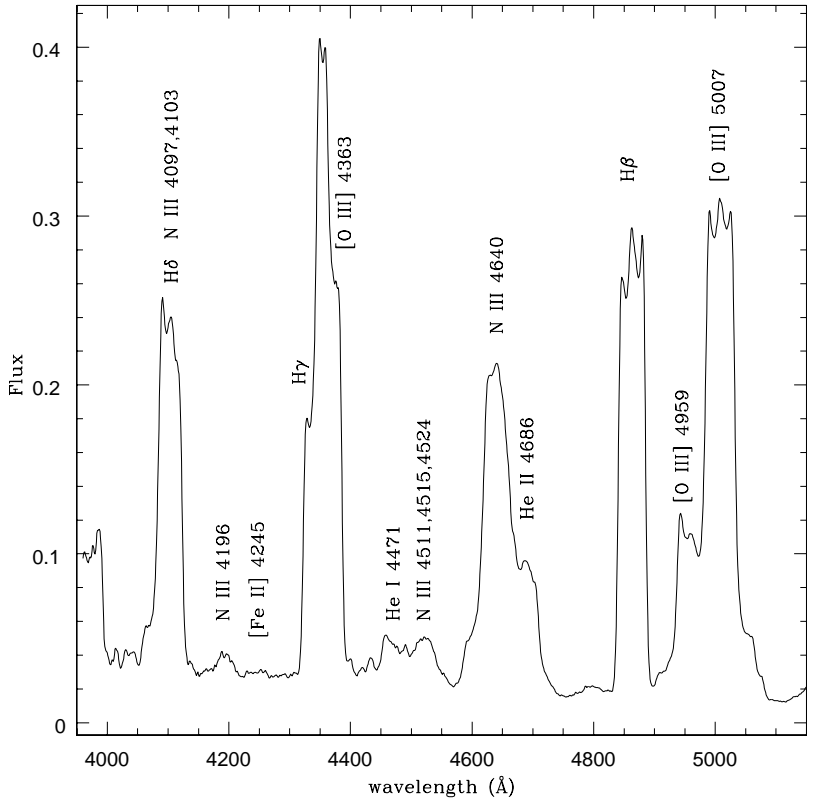

Fig. 10. A spectrum of V1494 Aql on Feb. 6, 2000. The unit of the ordinate is $10^{-10} \mathrm{erg} \mathrm{cm}^{-2} \mathrm{~s}^{-1} \AA^{-1}$.

December 17, and the latter is Na I D1+D2 $\lambda 5893$ blue-shifted by $-1260,-1270$, and $-1140 \mathrm{~km} \mathrm{~s}^{-1}$ on the respective days. It is, however, required a detailed work for the structure of the atmosphere of the nova to explain the reason why their radial velocities were so different from those of the main absorption components of the same lines.

\section{Transition stage and high velocity jets}

\subsection{Oscillation and spectral variation}

We were not able to observe the nova in January 2000 because of its low celestial location. When the first spectrum in the new observational season was taken on February 6, 2000, this nova was in the transition stage. A large oscillation of luminosity, which is a typical phenomenon of the transition stage, was observed in the period from February to the middle of April 2000 (JD 2451570-1650) as seen in Fig. 1.

The spectroscopic observations in the transition stage were made on February 6, 21, March 7, 16 and 17. Figure 10 shows a tracing of the spectrum on February 6 . The prominent emission lines are of H I, He I, He II, N II, N III, and [O III], while the emission lines of Fe II had disappeared. A weak emission line at $\lambda 4245 \AA$ is identified as [Fe II] $21 \mathrm{~F} \lambda 4244.8 \AA$. This nova may be one of rare fast novae which show [Fe II] lines like CP Pup (Weaver 1944). The other lines of [Fe II] are blended with stronger lines of other elements.

The first high dispersion spectrum with the Echelle spectrograph was taken on February 21, 2000, whose tracing is shown in Fig. 11. A part of the spectrum multiplied by ten is over-plotted as a dotted line to reveal the faint spectral features. There are prominent emission lines of $\mathrm{H} \mathrm{I}$, He I, He II, N II, N III, Si II, [O I], [O III], [N II], [Ca V] and [Fe VI]. The emission line at $\lambda 6086 \AA$ is usually identified as a blend of [Ca V] $\lambda 6085.9$ and [Fe VII] $\lambda 6085.5$. In this spectrum,
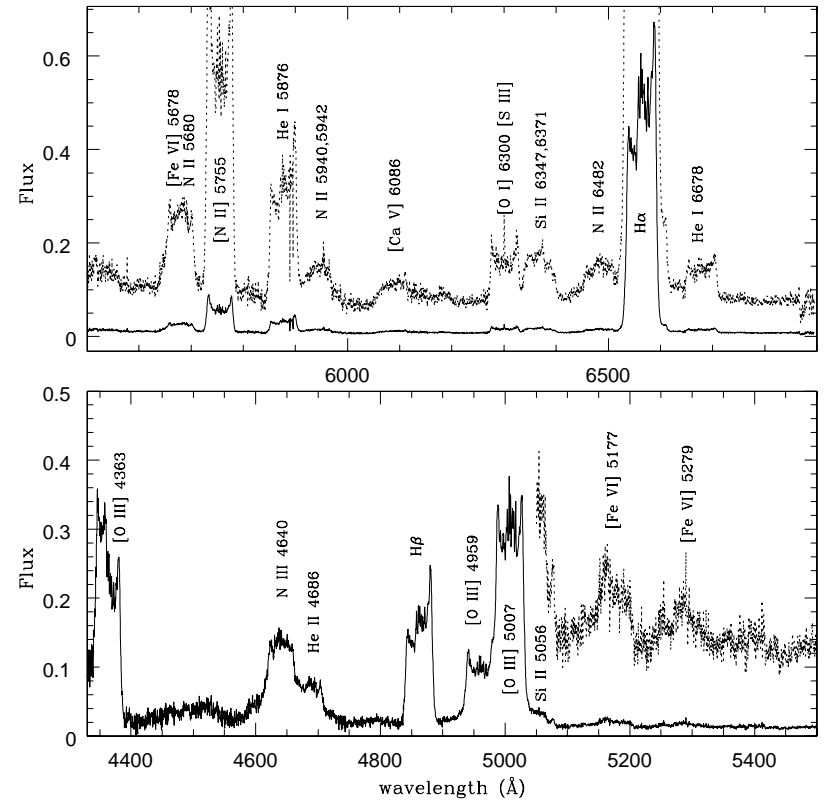

Fig. 11. A high dispersion spectrum of V1494 Aql on Feb. 21, 2000.

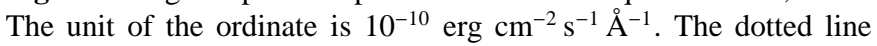
shows a tracing of the spectrum multiplied by ten.

however, the emission line may be due to [Ca V] $\lambda 6085.9$ alone, because no trace of [Fe VII] $\lambda 5721$ is seen. The emission lines of [Fe VII] were detected in September 2000 (Sect. 6.6). Intensities of prominent emission lines measured in the high dispersion spectra in the transition and the nebular stages are summarized in Table 4, and those measured in the medium dispersion spectra are given in Table 5. The effect of the interstellar extinction is corrected assuming $E(B-V)=0.6$. Detailed process of the decomposition of blended lines is given in Sect. 6.1. The observational errors of the intensities of the prominent emission lines are about $\pm 10 \%$, and those of the weak lines, which means that the intensity is of the order of $10^{-12} \mathrm{erg} \mathrm{cm}^{-2} \mathrm{~s}^{-1}$, may be more than $\pm 50 \%$. The intensities affected by larger errors are denoted by a colon.

During the transition stage four light maxima of the oscillation were observed at JD 2451587, 1606, 1621 and 1637 with an uncertainty of about \pm 1 day. The interval between the maxima was $16.5 \pm 1$ days and the mean amplitude of the oscillation was roughly $1.2 \mathrm{mag}$ in $V$ band. The observations on February 6, 21 and March 7 were made near light minima of the oscillation, while those on March 16 and 17 were made near the third maximum at JD 2451621.

Figures 12 and 13 show tracings of the high dispersion spectra taken on March 16 and 17, respectively. The spectral features near the light maximum were significantly different from those at light minima (Figs. 10 and 11). The emission complex at $\lambda 4640 \AA$ was already much stronger on March 16 (Fig. 12) than on February 21 (Fig. 11), and its further large growth (nitrogen flush) was observed on March 17 (Fig. 13). On the other hand, the emission line of He II 4686 weakened near the maximum of the oscillation and was embedded in the complex. The emission complex at $\lambda 4640 \AA$ showed two broad peaks on March 17 (Fig. 13), of which wavelengths were $\lambda 4610$ and $\lambda 4647 \AA$. The former may be due to N II $\lambda \lambda 4607.2$ 
Table 4. Intensities of selected emission lines corrected by $E(B-V)=0.6$ in the transition and nebular stages measured with the echelle spectrograph.

\begin{tabular}{|c|c|c|c|c|c|c|c|c|}
\hline \multirow[t]{2}{*}{ Elements } & \multicolumn{8}{|c|}{$I(\lambda)$} \\
\hline & Feb. 21 & Mar. 16 & Mar. 17 & Apr. 23 & Apr. 26 & May 15 & June 9 & Sep. 16 \\
\hline JD $2451000+$ & 595.7 & 619.7 & 620.7 & 657.6 & 660.6 & 679.6 & 704.6 & 804.4 \\
\hline [O III] 4363 & 6.5 & 5.7 & 4.2 & 4.5 & 4.3 & 4.3 & 3.5 & 0.92 \\
\hline He I 4471 & 0.30 & 0.54 & 1.1 & 0.14 & 0.16 & 0.15 & & \\
\hline N III 4511, 4515, 4524 & 0.58 & 0.40 & 2.8 & 0.31 & 0.22 & 0.23 & 0.40 & \\
\hline N II 4607, 4614 & 0.88 & & & 0.60 & 0.35 & 0.36 & 0.64 & 0.15 \\
\hline N II+N III & & $12.5 \dagger$ & $21.4 \dagger$ & & & & & \\
\hline N III 4641, 4634 etc. & 3.9 & & & 2.1 & 1.6 & 1.5 & 1.6 & 0.21 \\
\hline He II 4686 & 1.8 & $<0.02$ & $<0.02$ & 1.4 & 0.86 & 0.87 & 1.34 & 0.19 \\
\hline $\mathrm{H} \beta$ & 5.6 & 5.1 & 5.6 & 2.5 & 2.1 & 1.9 & 1.7 & 0.25 \\
\hline [Fe VII] 4942 & & & & & & & & $0.1:$ \\
\hline [O III] 4959 & 3.3 & 4.8 & 3.8 & 3.1 & 3.0 & 3.1 & 2.9 & 1.2 \\
\hline [O III] 5007 & 11.0 & 14.7 & 11.2 & 9.5 & 9.2 & 9.7 & 8.8 & 3.4 \\
\hline Si II 5056 & 0.28 & & & 0.09 & 0.08 & 0.08 & & \\
\hline$[\mathrm{Fe} \mathrm{VI}] 5147$ & 0.14 & & & 0.11 & 0.09 & 0.08 & 0.10 & 0.05 \\
\hline [Fe VI] 5177, N II 5180 & 0.26 & 0.35 & $0.4:$ & 0.23 & 0.17 & 0.17 & 0.17 & 0.03 \\
\hline$[\mathrm{Fe} \mathrm{VI}]$ 5279, [Fe III] 5270 & 0.24 & 0.13 & $0.6:$ & 0.07 & 0.12 & 0.10 & 0.08 & 0.08 \\
\hline$[\mathrm{Ca} \mathrm{V}] 5309$ & & & & 0.15 & 0.13 & 0.09 & 0.10 & 0.06 \\
\hline He II 5411 & $0.1:$ & & & 0.10 & 0.08 & 0.07 & 0.12 & 0.02 \\
\hline N II 5535 & $0.12:$ & 0.40 & 1.04 & 0.04 & 0.04 & 0.04 & & \\
\hline [Ca VII] 5616 & & & & & & & & 0.02 \\
\hline N II 5667 & 0.26 & 0.32 & 0.6 : & 0.11 & & & & \\
\hline N II 5680, [Fe VI] 5678 & 0.26 & 0.57 & 0.6 : & 0.21 & $0.23 *$ & $0.24 *$ & $0.25^{*}$ & $0.03 *$ \\
\hline [Fe VII $] 5721$ & & & & & & & & 0.12 \\
\hline [N II $] 5755$ & 2.5 & 3.2 & 5.0 & 1.14 & 1.24 & 1.05 & 0.76 & 0.15 \\
\hline He I 5876 & 1.13 & 1.50 & 1.27 & 0.47 & 0.35 & 0.28 & 0.26 & 0.02 \\
\hline N II 5940, 5942 & 0.28 & 0.20 & 0.38 & 0.08 & 0.06 & 0.07 & 0.06 & \\
\hline$[\mathrm{Ca} \mathrm{V}] 6086$ & 0.28 & & & 0.27 & 0.21 & 0.22 & 0.22 & $0.19 * *$ \\
\hline N II 6168 & 0.11 & 0.17 & 0.13 & & & & & \\
\hline uid. $\lambda 6200$ & & & & & & & & 0.02 \\
\hline [O I] 6300, [S III] 6310 & 0.46 & 0.13 & & 0.05 & 0.12 & $0.15:$ & 0.03 & 0.02 \\
\hline Si II 6347, 6371 & 0.48 & 0.17 & & 0.18 & 0.19 & & 0.05 & \\
\hline$[\mathrm{Fe} \mathrm{X}] 6375$ & & & & & & & & $0.06 \ddagger$ \\
\hline N II 6482 & 0.18 & & & 0.14 & 0.08 & 0.11 & 0.07 & 0.03 \\
\hline $\mathrm{H} \alpha$ & 24.8 & 26.4 & 26.9 & 11.4 & 8.2 & 6.9 & 6.0 & 0.92 \\
\hline [N II] 6584 & 0.49 & 0.63 & 0.62 & 0.33 & 0.28 & 0.26 & 0.21 & 0.07 \\
\hline He I 6678 & 0.31 & 0.30 & 0.38 & 0.17 & 0.11 & 0.11 & 0.11 & \\
\hline $\mathrm{H} \alpha / \mathrm{H} \beta$ & 4.4 & 5.2 & 4.8 & 4.6 & 3.9 & 3.7 & 3.5 & 3.7 \\
\hline$F W H M$ of $\mathrm{H} \beta$ & 2730 & 2740 & 2710 & 2720 & 2710 & 2700 & 2720 & 2690 \\
\hline
\end{tabular}

Unit of $I(\lambda)$ is $10^{-10} \mathrm{erg} \mathrm{cm}^{-2} \mathrm{~s}^{-1}$ and that of $F W H M$ is $\mathrm{km} \mathrm{s}^{-1}$.

$\dagger:$ N II $\lambda 4607$ and N III $\lambda 4641$ can not be separated.

*: blended with N II $\lambda 5667, * *$ : blended with [Fe VII] $\lambda 6086$, $\neq$ : blended with Si II $\lambda \lambda 6347,6371$.

and 4613.9, while the latter may be a blend of $\mathrm{N}$ III $\lambda \lambda 4634.2$, 4640.6, 4641.9, C III $\lambda \lambda 4647.4,4650.2,4651.4$ and probably O II $\lambda 4649.1 \AA$. In the red parts of the spectra the emission lines of N II appeared or strengthened at light maximum, hence He II and [Ca V] disappeared (Figs. 12 and 13). Similar spectral variations were known on the other oscillating novae GK Per and V603 Aql (see, e.g. McLaughlin 1943).

The intensities of the forbidden lines also varied according to the oscillation. McLaughlin (1943) reported that the intensity of [N $\mathrm{II}] \lambda 5755$ increased and that of [O III] $\lambda 4363$ decreased at light maxima of the oscillation of V603 Aql. Similar variations were seen on the present nova (Table 4). The intensity ratios of [O III] $I(5007+4959) / I(4363)$ and $[\mathrm{N} \mathrm{II}] I(6584+6548) / I(5755)$ suggest that the emitting region of $[\mathrm{N} \mathrm{II}]$ lines had higher temperature or higher density than that of [O III] lines at the maximum of the oscillation. (Detailed arguments of these intensity ratios are given in Sect. 6.2.) 
Table 5. Intensities of selected emission lines corrected by $E(B-V)=$ 0.6 in the transition and nebular stages measured with the $\mathrm{B} \& \mathrm{C}$ spectrograph.

\begin{tabular}{|c|c|c|c|}
\hline \multirow[t]{2}{*}{ Elements } & \multicolumn{3}{|c|}{$I(\lambda)$} \\
\hline & Feb. 6 & Mar. 7 & Sep. 9 \\
\hline JD $2451000+$ & 580.7 & 610.7 & 797.3 \\
\hline [Fe VI] 4072, [S II] 4076 & 0.95 & 0.64 & 0.04 \\
\hline $\mathrm{H} \delta, \mathrm{N}$ III 4097, 4103 & 6.0 & 3.7 & 0.36 \\
\hline N III 4196, 4200, He II 4200 & 0.30 & 0.17 & 0.04 \\
\hline$[\mathrm{Fe}$ II $] 4245$ & 0.10 & & \\
\hline $\mathrm{H} \gamma$ & 4.8 & 3.2 & 0.22 \\
\hline [O III] 4363 & 8.1 & 9.9 & 2.2 \\
\hline He I 4471 & 0.76 & 0.46 & 0.03 \\
\hline N III 4511, 4515, 4524 & 0.78 & 0.54 & 0.12 \\
\hline N II 4607 & 1.5 & 0.98 & 0.22 \\
\hline N III 4634, 4641, 4642 & 7.0 & 4.3 & 0.37 \\
\hline He II 4686 & 2.2 & 1.5 & 0.39 \\
\hline S II 4792 & 0.04 & 0.03 & \\
\hline N II 4803 & 0.07 & 0.03 & \\
\hline $\mathrm{H} \beta$ & 12.3 & 6.0 & 0.45 \\
\hline [O III] 4959 & 4.1 & 4.6 & 2.5 \\
\hline [O III] 5007 & 11.9 & 13.6 & 6.8 \\
\hline S II 5056 & 0.53 & 0.29 & 0.02 \\
\hline$[\mathrm{Fe} \mathrm{VI}] 5147$ & & & 0.04 \\
\hline$[\mathrm{Fe} \mathrm{VI}] 5177$ & & & 0.08 \\
\hline [Fe VII] 5721 & & & 0.20 \\
\hline [N II] 5755 & & & 0.35 \\
\hline He I 5876 & & & 0.04 \\
\hline [Fe VII] 6086, [Ca V] 6086 & & & 0.24 \\
\hline uid. $\lambda 6200$ & & & 0.02 \\
\hline [O I] 6300, [S III] 6310 & & & 0.05 \\
\hline [Fe X] 6375, Si II 6347 & & & 0.10 \\
\hline N II 6482 & & & 0.03 \\
\hline $\mathrm{H} \alpha$ & & & 1.40 \\
\hline [N II] 6584 & & & 0.13 \\
\hline He I 6678 & & & 0.01 \\
\hline C II 6750 & & & 0.01 \\
\hline$[\mathrm{A} \mathrm{V}] 7006$ & & & 0.03 \\
\hline He I 7065 & & & 0.02 \\
\hline [A III $] 7136$ & & & 0.03 \\
\hline [A IV] 7236, C II 7231, 7236 & & & 0.01 \\
\hline [O II $] 7319,7330$ & & & 0.18 \\
\hline $\mathrm{H} \alpha / \mathrm{H} \beta$ & & & 3.1 \\
\hline$F W H M$ of $\mathrm{H} \beta$ & 2790 & 2790 & 2820 \\
\hline
\end{tabular}

Unit of $I(\lambda)$ is $10^{-10} \mathrm{erg} \mathrm{cm}^{-2} \mathrm{~s}^{-1}$ and that of $F W H M$ is $\mathrm{km} \mathrm{s}^{-1}$.

The profiles of N II $\lambda 6167.8$ and [N II] $\lambda 5755$ on March 16 are shown in Fig. 14. The emission line of [N II] $\lambda 5755$ shows well separated blue and red peaks and some narrow smaller peaks around line center. Many prominent emission lines show similar profiles (Figs. 12 and 13). On the other hand, the emission line of N II $\lambda 6167.8$ shows only a featureless broad single peak. Also the emission lines of N III may have had similar hazy profiles near the light maximum, because no discrete peak was seen on the emission complex at $\lambda 4640 \AA$ (Figs. 12 and 13). The emission lines of N II and N III seem to have been
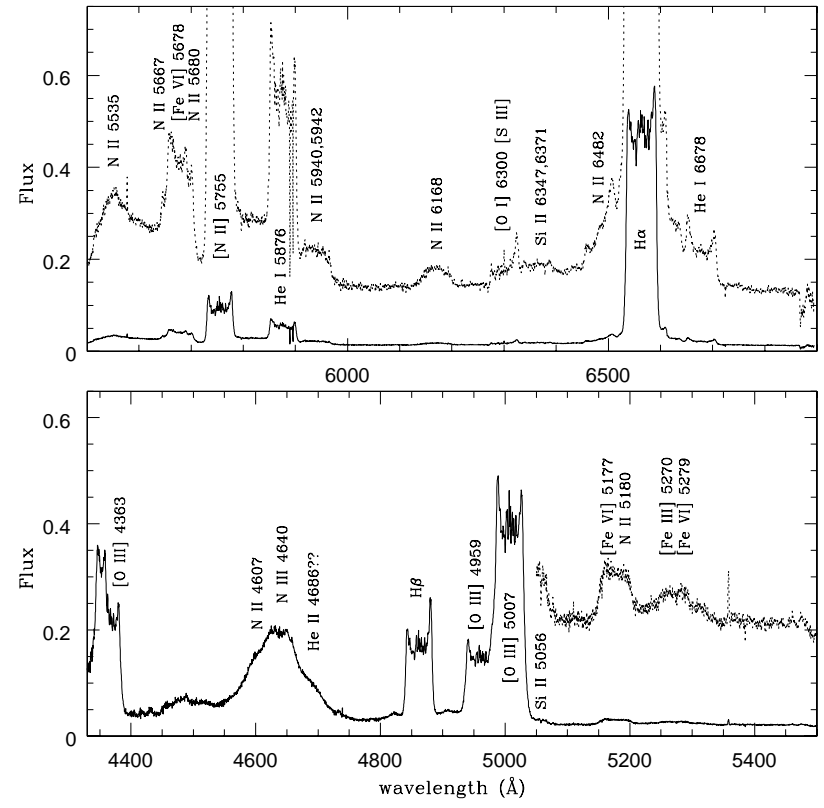

Fig. 12. A high dispersion spectrum of V1494 Aql on March 16, 2000. The unit of the ordinate is $10^{-10} \mathrm{erg} \mathrm{cm}^{-2} \mathrm{~s}^{-1} \AA^{-1}$.
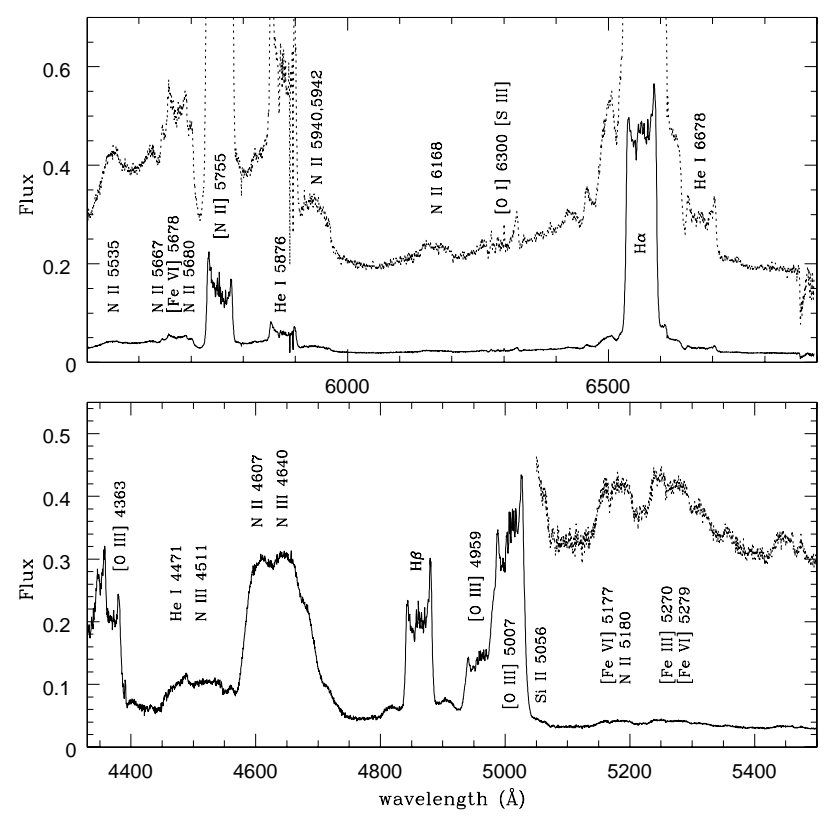

Fig. 13. A high dispersion spectrum of V1494 Aql on March 17, 2000. The unit of the ordinate is $10^{-10} \mathrm{erg} \mathrm{cm}^{-2} \mathrm{~s}^{-1} \AA^{-1}$.

formed in a more compact and nearly spherically symmetric region.

The emission component of $\mathrm{H} \beta$ of $\mathrm{V} 603 \mathrm{Aql}$ at minimum of the oscillation in the transition stage showed two well separated peaks, whereas a broad single peak, which is rather similar to that of our N II 16167.8 , was seen at maximum (McLaughlin 1956). Such a large variation of the profiles of $\mathrm{H} I$ lines was not observed in the present nova (Figs. 11-13). 


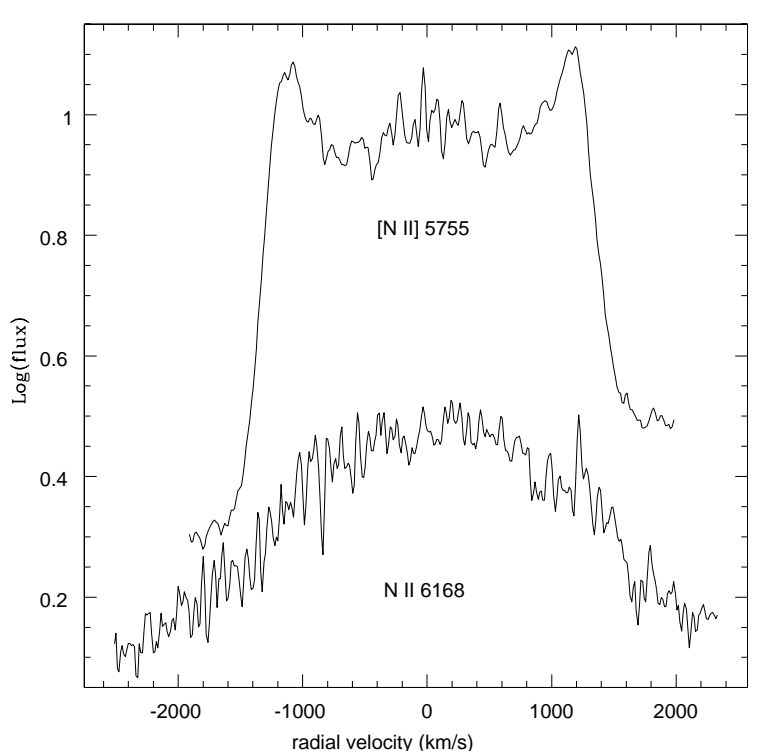

Fig. 14. Profiles of [N II] $\lambda 5755$ and N II $\lambda 6168$ on March 16, 2000. The ordinate is an arbitrary logarithmic scale.

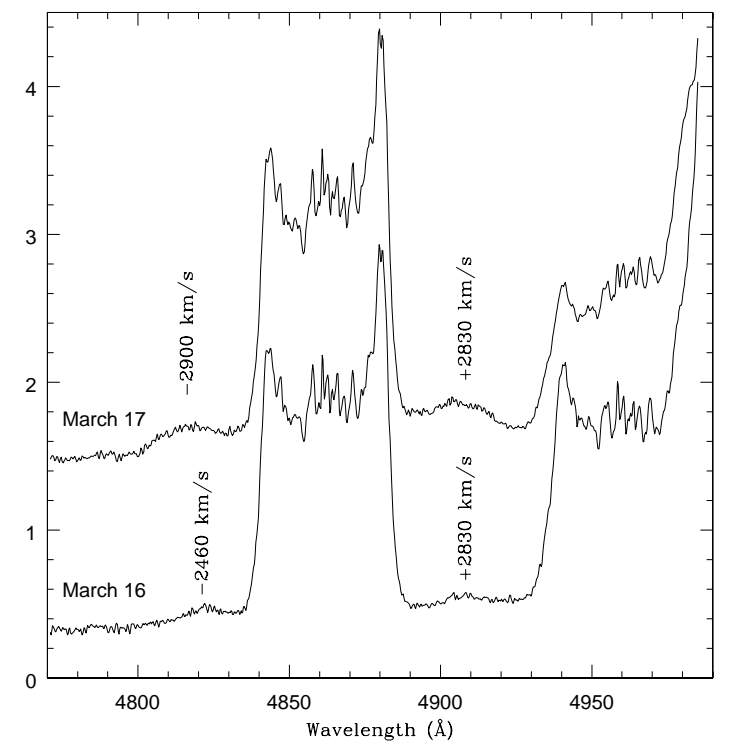

Fig. 15. High velocity emission wings of $\mathrm{H} \beta$ on March 16 and 17.

\subsection{High velocity jets}

High velocity broad emission components appeared in the blue and red sides of $\mathrm{H} \alpha$ and $\mathrm{H} \beta$ on March 16 and 17 (Figs. 12 and 13), namely near the light maximum of the oscillation. Figure 15 shows tracings of the $\mathrm{H} \beta$ region of the spectra taken on March 16 and 17. The radial velocities of the broad emission components in the frame of the nova system are given in Table 6 . The observational errors are about $\pm 100 \mathrm{~km} \mathrm{~s}^{-1}$. The radial velocity of the red component of $\mathrm{H} \alpha$ was not measured, because it was blended with the emission line of [N II] $\lambda 6584$. The sum of the flux of the broad emission components of $\mathrm{H} \beta$ was roughly $3 \%$ of that of the main emission component on March 16 and was about $6.5 \%$ on March 17 .

It seems that high velocity jets were ejected at the light maximum of the oscillation. At far as we know, however, no
Table 6. High velocity emission components of H I lines.

\begin{tabular}{lcc}
\hline \hline & $\begin{array}{c}\text { March 16 } \\
\left(\mathrm{km} \mathrm{s}^{-1}\right)\end{array}$ \\
\hline $\mathrm{H} \beta$ blue & -2460 & -2900 \\
$\mathrm{H} \beta$ red & +2830 & +2830 \\
$\mathrm{H} \alpha$ blue & -2590 & -2860 \\
\hline
\end{tabular}

previous nova showed a similar phenomenon during the transition stage. Even in the detailed description of the spectral variations of oscillating novae in the transition stage (McLaughlin 1943), such a high velocity emission component was never mentioned.

Rather similar high velocity emission components of $\mathrm{H}$ I and $\mathrm{He}$ II lines have been observed in super-soft Xray sources, e.g. RX J0513.9-6951 (Crampton et al. 1996) and RX J0019.8+2156 (Becker et al. 1998). The radial velocities of the high velocity emission components of the former object were $\pm 3600 \mathrm{~km} \mathrm{~s}^{-1}$ (Crampton et al. 1996) and those of the latter were about $\pm 800-900 \mathrm{~km} \mathrm{~s}^{-1}$ (Becker et al. 1998). The high velocity emission components of these objects, however, lasted at least several months keeping nearly the same velocities. On the other hand, the intensity and radial velocity of the high velocity emission components of the present nova were rapidly variable.

At the present time, our knowledge of the high velocity emission components of classical novae seems to be too poor to understand their properties. Detailed observations of the spectral variations in transition stage of novae in the future are required.

\section{Nebular stage}

The oscillation of luminosity in the transition stage terminated at the middle of April 2000 (Fig. 1). A tracing of the high dispersion spectrum taken on April 23 is shown in Fig. 16. Notable differences with respect to the spectrum on February 21 (Fig. 11) are growth of [O III] and [Ca V] lines relative to $\mathrm{H} \mathrm{I}$.

\subsection{Decomposition of blended lines}

Almost all prominent emission lines showed saddle-shaped profiles in the transition (Figs. 10-13) and nebular (Fig. 16) stages. Therefore, it is not possible to decompose blended lines with the usual Gaussian fitting. In this paper, we estimated the intensities of individual components of blended lines with the following method.

Since many lines in a spectrum showed nearly the same profile, we used the profile of $\mathrm{H} \beta$ as the standard, and measured the intensities in the blue and red quarters of the emission component. For example, the wavelengths of the two peaks of $\mathrm{H} \beta$ on the spectrum shown in Fig. 16 are $\lambda 4843.5$ and $\lambda 4880.0 \AA$, respectively. The full width at zero intensity of the emission component is about 1.4 times larger than the separation of the two peaks, that is the wavelengths at zero intensity are $\lambda 4836.2$ and $4887.3 \AA$. We measured the intensities in the blue quarter 

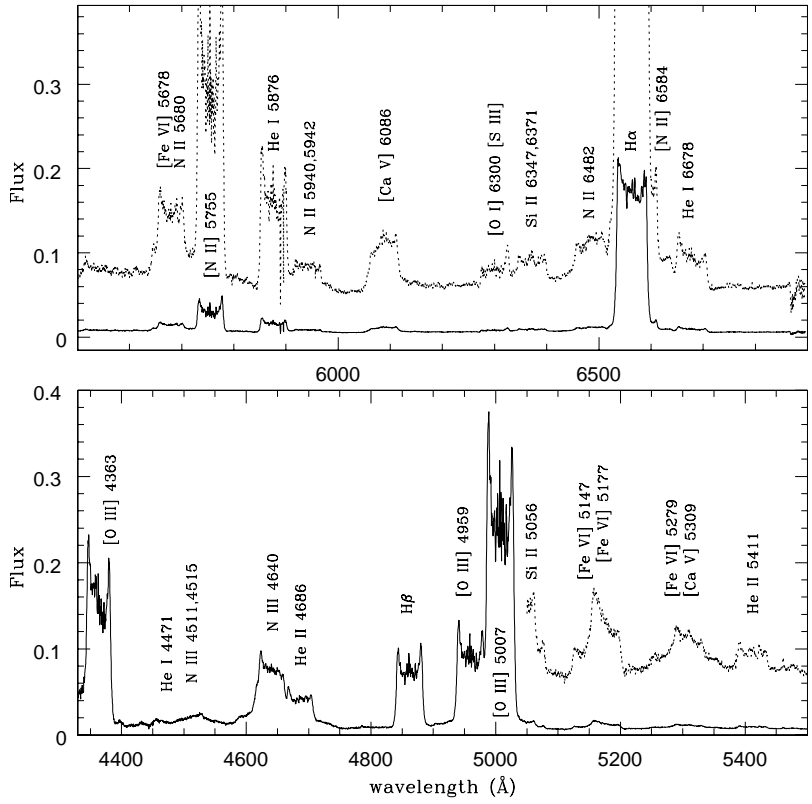

Fig. 16. A high dispersion spectrum of V1494 Aql on April 23, 2000. The unit of the ordinate is $10^{-10} \mathrm{erg} \mathrm{cm}^{-2} \mathrm{~s}^{-1} \AA^{-1}$. The dotted line shows a tracing of the spectrum multiplied by ten.

$\lambda \lambda 4836.2-4849.0$ and in the red quarter $\lambda \lambda 4874.5-4887.3$ of the emission component, then compared them with the total intensity. The ratio of the intensity in the blue quarter to the total one is 0.23 and that in the red quarter is 0.24 in the present case. Fortunately, the blue or red quarter of many blended lines were free from blending. In such cases, we were able to estimate the intensities of individual components of blended lines by this method. This method was not applied to N II and N III lines in the transition stage, because their profiles were different to that of $\mathrm{H} \beta$ (Figs. 12-14). The intensities of the prominent emission lines in the transition and nebular stages are given in Tables 4 and 5 .

\subsection{Electron temperature and density of ejecta}

As mentioned in Sect. 4.2, the high intensity ratio of $\mathrm{H} \alpha / \mathrm{H} \beta$ lasted during the transition stage, then gradually decreased (Fig. 8). The ratio $\mathrm{H} \alpha / \mathrm{H} \beta$ observed on April 26 and later (Tables 4 and 5) is rather close to those expected in the recombination theory (Hummer \& Storey 1987). Probably, the effect of the self-absorption (Netzer 1975) became weak in that period. The properties of the ejecta, therefore, are analysed using the spectra taken on and later than April 26, 2000.

Figure 17 shows the profiles of the emission lines of $\mathrm{H} \beta$, [N II] $\lambda 5755$ and [O III] $\lambda 5007$ on May 15. As seen in the figure, these three lines have the same detailed structures. It seems that these lines were formed in nearby regions. The uniform electron temperature and density of the emitting regions of these lines, therefore, could be a good approximation for the ejecta of this nova in the nebular stage. If this is the case, it is easy to obtain the electron temperature and density from the intensity ratios of the nebular lines to the auroral lines of [O III]

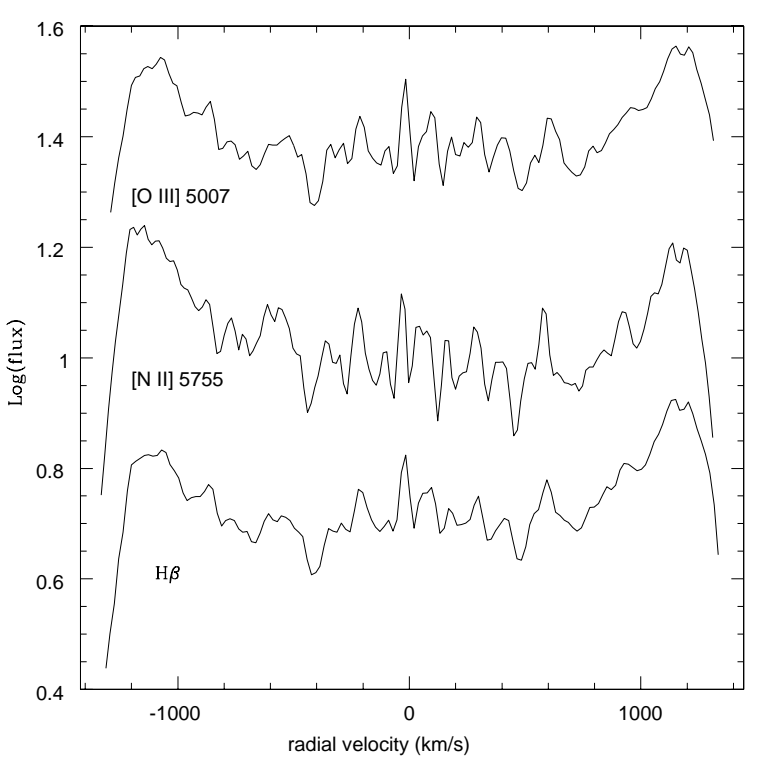

Fig. 17. Profiles of H $\beta$, [N II] 5755 and [O III] 5007 on May 15, 2000. The ordinate is an arbitrary logarithmic scale.

and [N II]. Seaton (1975) gave the following formulae.

$$
\begin{aligned}
\frac{I(5007+4959)}{I(4363)}= & 7.2 \exp \left(3.30 / t_{\mathrm{e}}\right) \frac{1+0.000054 x}{1+0.0635 x} \\
& {[\mathrm{~N} \mathrm{II}] } \\
\frac{I(6584+6548)}{I(5755)}= & 6.9 \exp \left(2.50 / t_{\mathrm{e}}\right) \frac{1+0.000057 x}{1+0.314 x},
\end{aligned}
$$

where $t_{\mathrm{e}}=T_{\mathrm{e}} / 10^{4}$ and $x=t_{\mathrm{e}}^{-1 / 2} N_{\mathrm{e}} / 10^{4}$. Since the emission line of [N II] $\lambda 6548$ was embedded in $\mathrm{H} \alpha$, its intensities were estimated assuming $I(6584) / I(6548)=2.94$ (Nussbaumer $\&$ Rusca 1979). The derived electron temperatures and densities are given in Table 7 . The errors in the temperatures are about $\pm 600 \mathrm{~K}$ and those in the densities are about $\pm 0.7 \times$ $10^{6}$ number $\mathrm{cm}^{-3}$.

The electron temperature was nearly constant at $10700 \mathrm{~K}$ during the nebular stage. The variation of the electron density with time is shown in Fig. 18. As seen in the figure, the electron density decreased smoothly with a decline rate of $N_{\mathrm{e}} \propto t^{-0.8}$, where $t$ is number of days from light maximum. This decline rate is much lower than that expected in the spherically symmetric expansion $N_{\mathrm{e}} \propto t^{-3}$. The ejecta might have a form of a ring and, probably, polar blobs such as those of V603 Aql (Weaver 1974), HR Del (Hutchings 1972), DQ Her (Martin 1989) etc.

Another possible cause of the low decline rate is a continuous mass outflow in the nebular stage. The model of Kato \& Hachisu (1994) shows that if the mass of the white dwarf is less than $1.15 M_{\odot}$, significant mass outflow will continue even more than 150 days after the explosion. Probably also this effect could contribute to the low decline rate.

As mentioned in Sect. 5.1, the intensity ratios of [O III] and [N II] lines at the light maximum of the oscillation in the transition stage were rather peculiar. If we assume that the electron temperature was $10700 \mathrm{~K}$ in the transition stage, the intensity ratios of the lines on March 16 and 17 give higher densities 
Table 7. Physical properties of ejecta.

\begin{tabular}{crrrrrrc}
\hline \hline Date & April 26 & May 15 & June 9 & Sep. 9 & Sep. 16 & mean & unit \\
days & 144.7 & 163.7 & 188.7 & 281.4 & 288.5 & & $\mathrm{~K}$ \\
\hline$T_{\mathrm{e}}$ & 10600 & 10700 & 10700 & 10900 & 10600 & $10700 \pm 100$ & $10^{6} \mathrm{~cm}^{-3}$ \\
$N_{\mathrm{e}}$ & 9.4 & 8.7 & 7.8 & 5.8 & 5.2 & & \\
$\mathrm{He} \mathrm{I} \mathrm{5876} \mathrm{C/R}$ & 0.25 & 0.26 & 0.26 & 0.28 & 0.25 & & \\
$N\left(\mathrm{He}^{+}\right) / N(\mathrm{H})$ & 0.10 & 0.09 & 0.09 & 0.05 & 0.05 & & \\
$N\left(\mathrm{He}^{++}\right) / N(\mathrm{H})$ & 0.04 & 0.04 & 0.07 & 0.08 & 0.07 & & \\
$N(\mathrm{He}) / N(\mathrm{H})$ & 0.14 & 0.13 & 0.16 & 0.13 & 0.12 & $0.13 \pm 0.01$ & \\
$m(\mathrm{H})$ & 4.7 & 4.6 & 4.7 & 1.5 & 1.0 & $4.7 \pm 1.0$ & $10^{-5} M_{\odot}$ \\
$m($ total $)$ & 6.2 & 6.0 & 6.3 & 1.9 & 1.3 & $6.2 \pm 1.4$ & $10^{-5} M_{\odot}$ \\
\hline
\end{tabular}

The means of $m(\mathrm{H})$ and $m$ (total) are those of April, May and June.

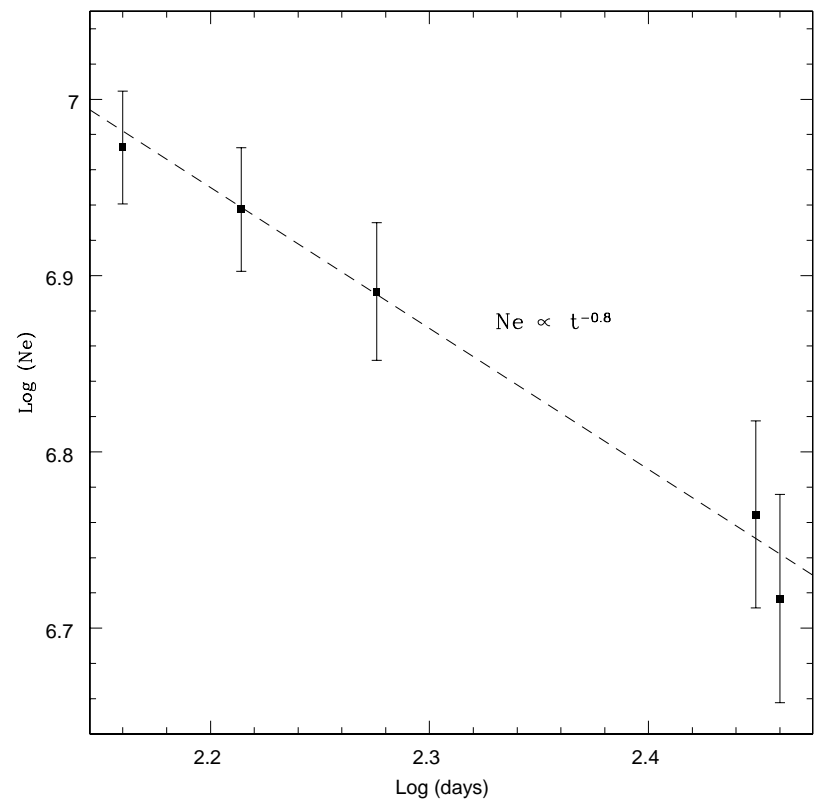

Fig. 18. Electron density of the ejecta in the nebular stage.

in the emission region of $[\mathrm{N} \mathrm{II}]$ than in the $[\mathrm{O} \mathrm{III}]$ region. On the other hand, if we extrapolate the decline rate of the electron density in the nebular stage to the transition stage, the same intensity ratios give higher temperature in the $[\mathrm{N}$ II] region than in the [O III] region. In any case, the condition of the ejecta at the light maximum of the oscillation may have been different from the thermal equilibrium.

\subsection{Helium abundance}

The helium abundance of the ejecta is estimated from the intensity ratios of He I $5876 / \mathrm{H} \beta$ and $\mathrm{He}$ II $4686 / \mathrm{H} \beta$.

In the previous sub-section, we have found $N_{\mathrm{e}}>5 \times$ $10^{6} \mathrm{~cm}^{-3}$ and $T_{\mathrm{e}} \cong 10700 \mathrm{~K}$ for the ejecta in the nebular stage. The effect of collisional excitation of He I 5876 can not be neglected in such a nebulosity (Clegg 1987; Peimbert \& Torres-Peimbert 1987). Here, we use the formula of Peimbert \& Torres-Peimbert (1987) to estimate the ratio of collision/recombination of He I 5876. The results are given as $\mathrm{He}$ I $5876 \mathrm{C} / \mathrm{R}$ in Table 7. The effective recombination coefficients of $\mathrm{H} \beta$, He I 5876 and He II 4686 at $T_{\mathrm{e}}=10700 \mathrm{~K}$ and $N_{\mathrm{e}}=8 \times 10^{6} \mathrm{~cm}^{-3}$ are:

$\alpha_{\mathrm{H} \beta}^{\mathrm{eff}}=2.97 \times 10^{-14} \mathrm{~cm}^{3} \mathrm{~s}^{-1}$

$\alpha_{\mathrm{HeI} 5876}^{\text {eff }}=4.74 \times 10^{-14} \mathrm{~cm}^{3} \mathrm{~s}^{-1}$

$\alpha_{\mathrm{He} \text { II } 4686}^{\text {eff }}=3.16 \times 10^{-14} \mathrm{~cm}^{3} \mathrm{~s}^{-1}$.

These values are obtained making interpolations from the results of Brocklehurst (1972) and Hummer \& Storey (1987). Using these values we have,

$\frac{N\left(\mathrm{He}^{+}\right)}{N(\mathrm{H})}=0.76 \frac{I(\mathrm{He} \mathrm{I} 5876)}{I(\mathrm{H} \beta)}$
$\frac{N\left(\mathrm{He}^{++}\right)}{N(\mathrm{H})}=0.090 \frac{I(\mathrm{He} \mathrm{II} \mathrm{4686)}}{I(\mathrm{H} \beta)}$.

If the electron temperature and density of the nebulosity are $T_{\mathrm{e}}=10700 \mathrm{~K}$ and $N_{\mathrm{e}}=5 \times 10^{6} \mathrm{~cm}^{-3}$, the numerical factors in the equations are 0.75 and 0.089 , respectively. The derived helium abundances are given in Table 7 . The errors in the individual values are about \pm 0.02 . Taking an average, we adopt the helium abundance of this nova as $N(\mathrm{He}) / N(\mathrm{H})=0.13 \pm 0.01$. The result on June 9 is not included in the average, because it is larger than the others. It is difficult to decide from our observations whether the helium abundance really increased on June 9. A similar temporal increasing of helium abundance, however, was observed on Nova Cen 1986 during the nebular stage (De Freitas Pacheco et al. 1989).

\subsection{Mass of ejecta}

Following Osterbrock (1989), we have a relation between the intensity of the $\mathrm{H} \beta$ emission line and the mass of hydrogen in the emitting nebula of pure hydrogen as:

$m(\mathrm{H}) / M_{\odot}=\frac{D^{2}}{N_{\mathrm{e}}} \frac{2.455 \times 10^{-2}}{\alpha_{\mathrm{H} \beta}^{\mathrm{eff}}} I(\mathrm{H} \beta)$,

where $m(\mathrm{H})$ is the mass of the hydrogen in the nebula, $D$ is distance to the object in $\mathrm{kpc}, N_{\mathrm{e}}$ is electron density in number $\mathrm{cm}^{-3}$, and $I(\mathrm{H} \beta)$ is intensity of $\mathrm{H} \beta$ emission line in $\mathrm{erg} \mathrm{cm}^{-2} \mathrm{~s}^{-1}$. In the case of $T_{\mathrm{e}}=10700 \mathrm{~K}$ and $N_{\mathrm{e}}=8 \times$ $10^{6} \mathrm{~cm}^{-3}$ we have:

$m(\mathrm{H}) / M_{\odot}=\frac{D^{2}}{N_{\mathrm{e}}} 8.27 \times 10^{11} I(\mathrm{H} \beta)$, 
where the effective recombination coefficient obtained in the previous sub-section is used. The numerical factor in the equation is $8.30 \times 10^{11}$ in the case of $T_{\mathrm{e}}=10700 \mathrm{~K}$ and $N_{\mathrm{e}}=5 \times$ $10^{6} \mathrm{~cm}^{-3}$. If we take into account the abundance of helium, the electron density will be given as:

$N_{\mathrm{e}}=N(\mathrm{H})+N\left(\mathrm{He}^{+}\right)+2 N\left(\mathrm{He}^{++}\right)$.

The total mass of the nebula may be given as:

$$
\begin{aligned}
m(\text { total })= & m_{\mathrm{H}} V(N(\mathrm{H})+4 N(\mathrm{He})) \\
= & m(\mathrm{H})\left(1+4 \frac{N(\mathrm{He})}{N(\mathrm{H})}\right) /\left(1+\frac{N\left(\mathrm{He}^{+}\right)}{N(\mathrm{H})}\right. \\
& \left.+\frac{2 N\left(\mathrm{He}^{++}\right)}{N(\mathrm{H})}\right),
\end{aligned}
$$

where $m_{\mathrm{H}}$ is the mass of a hydrogen atom, $V$ is the volume of the nebula and $m(\mathrm{H})=m_{\mathrm{H}} N_{\mathrm{e}} V$. The derived masses of the ejecta are given in Table 7 , where we assumed the distance $D=1.6 \mathrm{kpc}$. The rapid decreasing of the mass of the ejecta in September 2000 may have been due to a decreasing of the number of ionizing photons for hydrogen atoms. The total mass of the ejecta may be $6.2 \pm 1.4 \times 10^{-5} M_{\odot}$.

If we assume that the ejecta has a uniform density, the mass of hydrogen in the ejecta will be given as:

$m(\mathrm{H})=\frac{4}{3} \pi R^{3} m_{\mathrm{H}} N_{\mathrm{e}} \epsilon$,

where $R$ is radius of the ejecta and $\epsilon$ is a factor that represents the filling factor of the ejecta and the geometrical covering factor.

The radius of the ejecta on April 26 may be about $3.6 \times$ $10^{10} \mathrm{~km}$, where we assumed the expansion velocity of the ejecta to be $2900 \mathrm{~km} \mathrm{~s}^{-1}$. Using the mass and the electron density given in Table 7, we have $\epsilon=0.030$ for April 26. The error in $\epsilon$ is mainly due to the uncertainty in the expanding velocity. If the error in the expanding velocity is $\pm 200 \mathrm{~km} \mathrm{~s}^{-1}$, that in $\epsilon$ is about \pm 0.01 . This factor was 0.022 on May 15 and 0.016 on June 9. These small factors also suggest that the ejecta was not spherically symmetric, but may have had a ring-like shape.

\subsection{An inclined ring}

The low decline rate of the electron density (Sect. 6.2) and the small factor $\epsilon$ obtained in the previous sub-section suggest that the ejecta may have had a ring-like form in the nebular stage. In addition to these results, the widths of the emission lines seem to support the model of the ring-like form of the ejecta.

The outflow velocities measured with the blue-shifts of the absorption lines in the early decline stage were larger than $2500 \mathrm{~km} \mathrm{~s}^{-1}$ (Fig. 9) and probably reached up to $2900 \mathrm{~km} \mathrm{~s}^{-1}$ (Sect. 4.3). If the ejecta were expanding with such velocities, the full widths of the emission lines in the nebular stage should have been about $5000-6000 \mathrm{~km} \mathrm{~s}^{-1}$. On the other hand, the FWHMs of the emission component of $\mathrm{H} \beta$ in the nebular stage were about $2700 \mathrm{~km} \mathrm{~s}^{-1}$ (Table 4) and the full width at zero intensity, which was measured on April 23 (Sect. 6.1), was $3200 \mathrm{~km} \mathrm{~s}^{-1}$. The widths of the other prominent emission lines were nearly the same.
The disagreement between the radial velocities of the absorption lines and the widths of the emission lines could be explained if we assume that the ejecta in the nebular stage formed a ring which was expanding with the velocity of $2900 \mathrm{~km} \mathrm{~s}^{-1}$, but was inclined by about 30 degrees from the line of sight.

At the present time, three years after the explosion, the radius of the ejecta may be of order of 0.1 arcsec. This radius is large enough to be measured from ground based instruments or by the Hubble Space Telescope. Further works are awaited.

\subsection{Highly excited lines}

A tracing of a red spectrum taken on September 9 is shown in Fig. 19, where the emission lines of [Fe VII] and the coronal line [Fe X] 16347 are seen. Almost all prominent emission lines in the optical region (this paper) and the coronal lines in the infrared region (Rudy et al. 2001) showed double-peaked profiles, but the profile of [Fe X] $\lambda 6374$ looks different. Its strange profile may be due to a blending with Si II 16347 . The $F W H M$ of $\mathrm{H} \beta$ was about $2700 \mathrm{~km} \mathrm{~s}^{-1}$ throughout the nebular stage (Table 4), and the widths of the other prominent emission lines were nearly the same. If the widths of Si II $\lambda 6347$ and [Fe X] $\lambda 6375$ were also about $2700 \mathrm{~km} \mathrm{~s}^{-1}$, the red part of Si II and the blue part of $[\mathrm{Fe} \mathrm{X}]$ should overlap each other producing a single strong peak between them. A similar profile of blended lines is seen in the case of [Fe VI] $\lambda \lambda 5147$ and 5177 on April 23 (Fig. 16)

Figure 20 shows a tracing of our last spectrum taken on September 16, 2000, where [O III] $\lambda 5007$ is much stronger than $\mathrm{H} \alpha$. Like the spectrum taken on September 9 (Fig. 19), some emission lines of [Fe VII] and the coronal line [Fe X] $\lambda 6347$ are seen. No other coronal line in the optical region, e.g. [Fe XIV] $\lambda 5304$, is seen in this spectrum. The same condition is known also in Nova (GQ) Mus 1983 (Krauter \& Williams 1989). On the other hand, many coronal lines in the infrared region, e.g. [Si VII], [Si X], [Ca VIII], [Al IX], [S IX] etc., were observed in July 2000 (Venturini et al. 2000) and July 2001 (Rudy et al. 2001).

The unidentified emission line at $\lambda 6200$, which was detected on CP Pup (Sanford 1945), is seen on this nova (Figs. 19 and 20). Since this line appeared in a later nebular stage, it may relate to a highly ionized ion. Its precise laboratory wavelength should be $\lambda 6199.5 \pm 0.5 \AA$.

\section{Concluding remarks}

In this paper, a new distance $1.6 \pm 0.2 \mathrm{kpc}$ is derived for the nova V1494 Aql. The mass of the ejecta is estimated as $6.2 \pm$ $1.4 \times 10^{-5} M_{\odot}$ and the helium abundance as $N(\mathrm{He}) / N(\mathrm{H})=$ $0.13 \pm 0.01$. The electron temperature lasted nearly constant at $10700 \mathrm{~K}$ during the nebular stage, at least until the end of our monitoring in September 2000. The electron density decreased as $N_{\mathrm{e}} \propto t^{-0.8}$, where $t$ is number of days from light maximum. This low decline rate suggests that the ejecta had a ring like form and that probably a significant mass outflow continued in the nebular stage.

This nova seems to be similar to V603 Aql in many aspects, e.g. the oscillation of luminosity in the transition stage, 


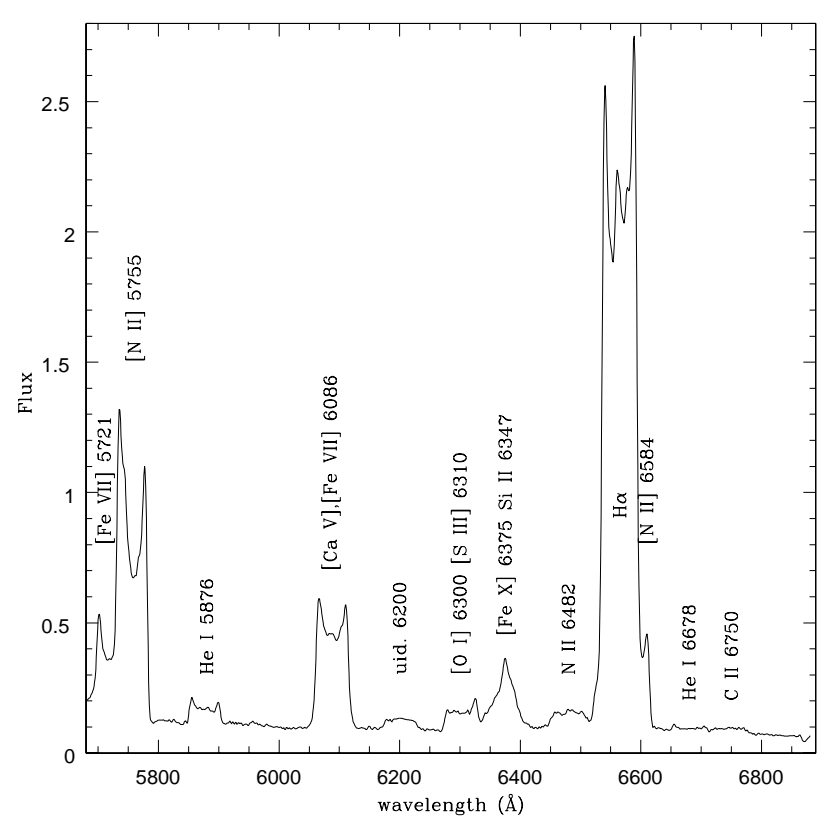

Fig. 19. A spectrum of V1494 Aql on September 9, 2000. The unit of the ordinate is $10^{-12} \mathrm{erg} \mathrm{cm}^{-2} \mathrm{~s}^{-1} \AA^{-1}$.
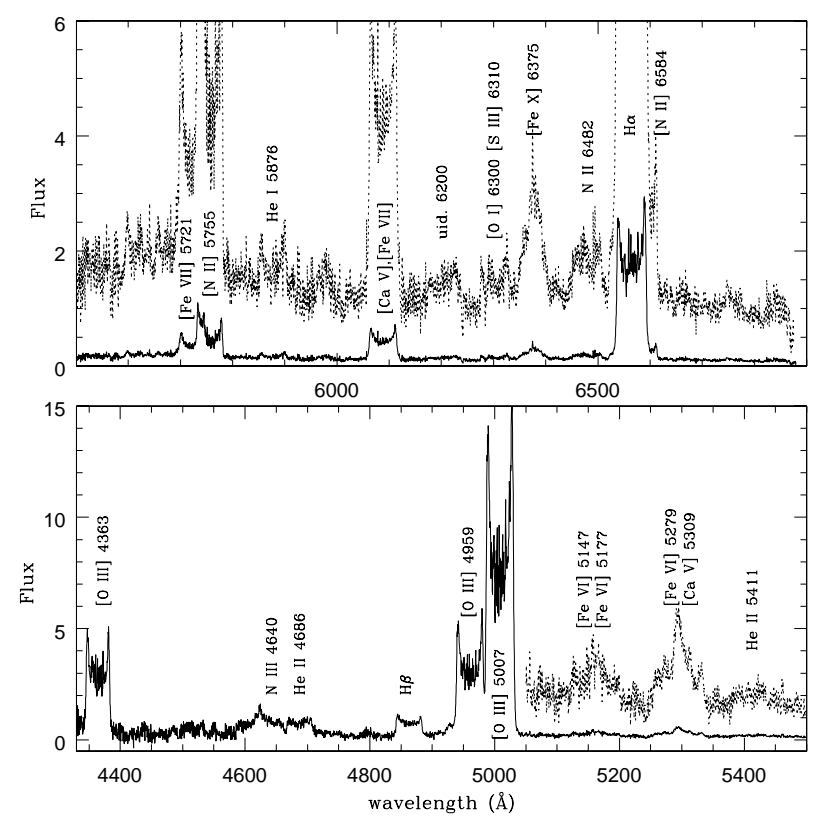

Fig. 20. A high dispersion spectrum of V1494 Aql on September 16, 2000. The unit of the ordinate is $10^{-12} \mathrm{erg} \mathrm{cm}^{-2} \mathrm{~s}^{-1} \AA^{-1}$.

the spectral variations and the form of the ejecta. However, the high velocity jets observed around the light maximum of the oscillation in the transition stage have not been found in any previous nova. Careful spectroscopic observations in the transition stages of other novae are required to confirm the existence and better define the properties of the high velocity jets.

Acknowledgements. We are grateful to Profs. I. Hachisu and M. Kato for the useful discussions and comments, and to Prof. R. Barbon for the careful reading of the manuscript and useful suggestions. Thanks are also due to Dr. T. Kato, Dr. D. Nogami, Mr. S. Kiyota, and many other professional and amateur astronomers who supplied photometric data of V1494 Aql on the VSNET. HHE gratefully thanks both Italian Government Ministry of Foreign Affairs and the Research Fund of the University of Istanbul for financial support under a scholarship and the project 1506/280700.

\section{References}

Anupama, G. C., Sahu, D. K., \& Mayya, Y. D. 2001, Bull. Astron. Soc. India, 29, 375

Becker, C. M., Remillard, R. A., Rappaport, S. A., \& McClintock, J. E. 1998, ApJ, 506, 880

Brocklehurst, M. 1972, MNRAS, 157, 211

Clegg, R. E. S. 1987, MNRAS, 229, 31p

Cohen, J. G. 1975, ApJ, 197, 117

Crampton, D., Hutchings, J. B., Cowley, A. P., et al. 1996, ApJ, 456, 320

de Freitas Pacheco, J. A., Dell'Aglio Dias da Costa, R., \& Codina, S. J. 1989, ApJ, 347, 483

Ferland, G. J. 1978, ApJ, 219, 589

Hummer, D. G., \& Storey, P. J. 1987, MNRAS, 224, 801

Hutchings, J. B. 1972, MNRAS, 158, 177

Jenkins, E. B., \& Savage, B. D. 1974, ApJ, 187, 243

Kato, M., \& Hachisu, I. 1994, ApJ, 437, 802

Kawabata, K. S., Akitaya, H., Hirakata, N., et al. 2001, ApJ, 552, 782

Kiss, L. L., \& Thomson, J. R. 2000, A\&A, 355, L9

Krautter, J., \& Williams, R. E. 1989, ApJ, 341, 968

McLaughlin, D. B. 1943, Publ. Obs. Univ. of Michigan, 8, 149

McLaughlin, D. B. 1956, PASP, 68, 444

McLaughlin, D. B. 1960, in Stellar Atmospheres, ed. J. L. Greenstein, (The University of Chicago Press), 585

Martin, P. G. 1989, in Classical Novae, ed. M. F. Bode, \& A. Evans (Chichester: J. Wiley \& S. Ltd.), 93

Moore, C. E. 1959, A Multiplet Table of Astrophysical Interest, US Dept. of Commerce, Office of Technical Services, Washington DC.

Münch, G. 1968, in Nebulae and Interstellar Matter, ed. B. M. Middlehurst, \& L. H. Aller (The University of Chicago Press), 365

Netzer, H. 1975, MNRAS, 171, 395

Nussbaumer, H., \& Rusca, C. 1979, A\&A, 72, 129

Osterbrock, D. E. 1989, Astrophysics of Gaseous Nebulae and Active Galactic Nuclei (Mill Valley: University Science Books)

Peimbert, M., \& Torres-Peimbert, S. 1987, Rev. Mex. Astron. Astrofis., 14, 540

Pereira, A. 1999, IAU Circ. 7323

Rudy, R. J., Mazuk, S., Venturini, C., Lynch, D. K., \& Mortfield, P. 2001, IAU Circ. 7674

Sanford, R. F. 1945, ApJ, 102, 357

Seaton, M. J. 1975, MNRAS, 170, 475

Venturini, C., Rudy, R. J., Lynch, D. K., Mazuk, S., Puetter, R. C., \& Armstrong, T. 2000, IAU Circ. 7490

Weaver, H. F. 1944, ApJ, 99, 280

Weaver, H. F. 1974, in Highlights of astronomy, 3, ed. G. Contopoulos (D. Reidel Pub. Co.), 509 